\title{
A comparison between size dependent paraelectric and ferroelectric BaTiO3 nanoparticle doped nematic and ferroelectric liquid crystals
}

DOI:

$10.1063 / 1.4976859$

\section{Document Version}

Accepted author manuscript

Link to publication record in Manchester Research Explorer

Citation for published version (APA):

Al-Zangana, S., Turner, M., \& Dierking, I. (2017). A comparison between size dependent paraelectric and ferroelectric BaTiO3 nanoparticle doped nematic and ferroelectric liquid crystals. Journal of Applied Physics, 121(8), [085105]. https://doi.org/10.1063/1.4976859

\section{Published in:}

Journal of Applied Physics

\section{Citing this paper}

Please note that where the full-text provided on Manchester Research Explorer is the Author Accepted Manuscript or Proof version this may differ from the final Published version. If citing, it is advised that you check and use the publisher's definitive version.

\section{General rights}

Copyright and moral rights for the publications made accessible in the Research Explorer are retained by the authors and/or other copyright owners and it is a condition of accessing publications that users recognise and abide by the legal requirements associated with these rights.

\section{Takedown policy}

If you believe that this document breaches copyright please refer to the University of Manchester's Takedown Procedures [http://man.ac.uk/04Y6Bo] or contact uml.scholarlycommunications@manchester.ac.uk providing relevant details, so we can investigate your claim.

\section{OPEN ACCESS}



$\mathrm{BaTiO}_{3}$ nanoparticle doped nematic and ferroelectric liquid crystals

\author{
S. Al-Zangana, ${ }^{1}$ M. Turner, ${ }^{2}$ and I. Dierking,,${ }^{1, a)}$ \\ ${ }^{1}$ School of Physics and Astronomy, University of Manchester, Oxford Road, Manchester M13 9PL, United Kingdom \\ ${ }^{2}$ School of Chemistry, University of Manchester, Oxford Road, Manchester M13 9PL, United Kingdom
}

The electro-optic and dielectric properties of barium titanate, $\mathrm{BaTiO}_{3}$, nanoparticle (NP) doped nematic and ferroelectric liquid crystal materials were examined with respect to different size and concentration of the NPs. Smaller size paraelectric NPs $\approx \approx 0 \mathrm{~nm})$ are compared to larger, ferroelectric NPs $(\approx 240 \mathrm{~nm})$. It is found that for concentrations larger than $0.5 \mathrm{vol} \%$ the ferroelectric NPs exhibit an increasing effect on the electro-optic response of the nematic liquid crystal, which is demonstrated by the enhancement of the dielectric anisotropy. This could be induced by the coupling of the electrical dipole moments in the spherical NPs with the LC director field. The electro-optical properties of the $\mathrm{SmC}^{*}$ phase, like tilt angle $\Theta$, switching time $\tau_{s}$ and spontaneous polarisation $P_{S}$ are found to be independent of the concentration and size of the NPs. The rotational viscosity $\eta$ calculated from response times, polarization reversal current and dielectric properties is comparable for all three methods employed and practically independent of particle concentration and size. The relaxation frequency $f_{R}$ of the Goldstone mode is higher in the ferroelectric NPs suspensions of $2.0 \mathrm{vol} \%$ as compared to the smaller paraelectric NPs.

\footnotetext{
a) Author to whom correspondence should be addressed. Electronic address: Ingo.dierking@manchester.ac.uk
} 


\section{PublishingINTRODUCTION}

Hybrid systems of nanoparticles (NPs) and liquid crystals (LCs) have appeared as a new area of research as there is the advantage of combining the order of LCs and the physical properties of NPs. In addition, LCs are particularly chosen due to their fluidity and unique anisotropic properties such as dielectric, refractive indices and elastic constants ${ }^{1-3}$ to exploit the possibilities of a switchable matrix. The main objectives behind investigating NP-LC hybrid systems are to tune the properties of the liquid crystal, ${ }^{4-8}$ to modify the properties of NPs through transfer of self-organisation in the liquid crystal medium, ${ }^{9}$ and/or to obtain additional functionalities not available by either materials in their intrinsic form.

Reznikov et al. have shown that a low concentration of ferroelectric NPs doped into a nematic liquid crystal reduces the threshold voltage, $\mathrm{V}_{\text {th }}$, to half its original value. ${ }^{6}$ It was later reported that the addition of $\mathrm{BaTiO}_{3}$ nanoparticles of less than $50 \mathrm{~nm}$ size to a nematic liquid crystal approximately doubles the dielectric anisotropy, $\Delta \varepsilon_{\text {Susp. }}^{\prime}{ }^{10}$ This is of predominant interest for LC device applications since any reduction of $\mathrm{V}_{\mathrm{th}}$ can lead to a reduction of the power consumption.

However, NP doped LC systems do not always result in improved physical and electro-optical properties. For instant, Blach et.al. ${ }^{8}$ and Fenghua et.al. ${ }^{5}$ showed that doping ferroelectric nanoparticles of $\mathrm{BaTiO}_{3}$ into 4-cyano-4'-pentylbiphenyl (5CB) decreased the Fréedericksz threshold $\mathrm{V}_{\text {th }}$ by approximately $30 \%$, while no changes of $\mathrm{V}_{\text {th }}$ were found by Glushchenko et $a l .{ }^{11}$ and according to Klein et $a l .{ }^{4}$ the nanoparticles even increased the treshold voltage. This could be attributed to a varied functionality of the NPs in the LC medium due to their physical and chemical properties.

The stability and dispensability of NPs in an isotropic medium have been a major challenge in colloid science. In liquid crystal based systems the instability of particle dispersions is further influenced by the order of the matrix, as well as the intrinsic properties of the particles (ferroelectric, ferromagnetic, etc.). In addition it is found that the distortion of the director field $\mathbf{n}(\mathbf{r})$ and the change in order parameter, S, depends on the size of dispersed NPs. Aggregation 
Publishiqugckly leads to phase separation which in turn strongly influences the properties of the material under investigation.

A reduction of the particle size of ferroelectric nanomaterials will generally lead to the eventual loss of ferroelectricity at small sizes. ${ }^{12}$ However, Basun et al. showed that the surface stress resulting from the milling technique sustains ferroelectricity even at a small sizes $(<10 \mathrm{~nm})^{13}$. Accordingly, the milling technique has extensively been utilised for ferroelectric NPs. ${ }^{6,14-22}$ In addition to the size, the nanoparticle surface properties are also crucial for the manifestation of a well dispersed medium. Surface modification of NPs has been mainly been employed to achieve long-term stability of NPs in the LC medium and to smoothen director distortions around NPs, ${ }^{23}$ thus reducing the cost of elastic energy. This is often achieved by coating the surface with monomolecular organic materials, surfactants (stabiliser), or ligands. For instance, oleic acid, ${ }^{7,10,17,20,21,24-27}$ selenium docosane ${ }^{28}$ and dodecylbenzenesulphonic acid ${ }^{29,30}$ have been widely utilized for the coating of NPs.

Recently, ferroelectric nanoparticle doped liquid crystals have become the subject of interest due to their potential in the applications relating to memory effects. ${ }^{24,31,32}$ These effects are achieved as the NPs are maintained in their switched state even after the removal of the electric field. Moreover, in the isotropic phase, the mesogens around individual nanoparticles gain orientational order, which is induced by the electric dipole moment of the NPs, thus producing a microscopic pseudo-nematic domain. The nematic to isotropic phase transition temperature $\mathrm{T}_{\mathrm{N}-\mathrm{I}}$ could be increased for an optimal concentration and well dispersed ferroelectric NPs. The wider the liquid crystal phase range, the higher the potential for display and other applications. In addition, the permanent electric dipole moment of the ferroelectric NPs could be electrically aligned with the direction of the director field or the applied electric field. This will significantly improve the electrooptic performance and especially the switching speed of so called "ferronematics".

While the effects of nanoparticles on LC host media have been studied widely, controversial results have so far been reported and little success was achieved to manifest novel functionalities 
Publishiøfgthe LC-particle dispersions. Thus, further systematic experimental investigations are required to determine how NPs influence LC properties.

We here compare the doping effects of two different sizes of nanoparticle barium titanate, BTO1 with approximately $80 \mathrm{~nm}$ diameter (paraelectric), and BTO2 with about 240nm diameter (ferroelectric) into nematic and ferroelectric SmC* liquid crystal phases. The electro-optic and dielectric properties were investigated in detail, also as a function of loading concentration. We use commercial liquid crystal materials $5 \mathrm{CB}$ and a ferroelectric Felix mixture, due to their known neat properties and wide use $e^{4,8,19,33,34}$ in research and applications.

\section{MATERIALS AND EXPERIMENTAL METHODOLOGY}

\subsection{SAMPLE PREPARATION}

Two different commercial liquid crystalline materials (i) nematic 4-cyano-4'-pentylbiphenyl (5CB), from SYNTHON Chemicals GmbH \& Co. KG, Germany, with phase sequence Cryst. 18 N 35.4 Iso., and (ii) a ferroelectric liquid crystal Felix M4851/050, from Clariant, Germany, with phase sequence Cryst. $-15 \mathrm{SmC}^{*} 66 \mathrm{SmA}^{*} 72.7 \mathrm{~N}^{*} 76$ Iso. were used for the investigations (all temperatures in ${ }^{\circ} \mathrm{C}$ ). $5 \mathrm{CB}$ is a single molecular component material. It has a large positive dielectric anisotropy of $\Delta \varepsilon^{\prime}=11.5$ at room temperature, due the cyano end group that gives rise to the electric dipole moment mainly along the long axis of the molecule. ${ }^{35}$ Felix M4851/050 is a multi-component ferroelectric LC mixture. Two different sizes of barium titanate nanopowders (US Research Nanomaterials, Inc) were employed. A 80nm batch, which is paraelectric and here abbreviated as BTO1 and a ferroelectric 240nm batch named BTO2.

Mixtures of different volume percentage (vol.\%) of NPs in both 5CB and Felix were prepared by the following procedure. The NPs and LCs were gently mixed using an agate mortar and pestle in order to reduce the size of any possible aggregates. Since the liquid crystals are both roomtemperature LC materials, this resulted in a uniform dispersion of nanoparticles within the LC, without any change of composition during the mixing process. For $5 \mathrm{CB}$, mixtures of $0.5,1.0$, 2.0, 4.0, 6.0 and 8.0vol\% were prepared at room temperature, while for Felix, the mixtures of 1.0 and $2.0 \mathrm{vol} \%$ were prepared at $70^{\circ} \mathrm{C}$ in the $\mathrm{SmA}^{*}$ phase. As a second method the NPs are 
Publishitidg sferred to the liquid crystals $5 \mathrm{CB}$ and Felix by use of organic solvents isopropanol and dichloromethane, respectively. Stable dispersions were achieved by applying sonication for 20 minutes, which was examined by dynamic light scattering (DLS). Different samples $\mathrm{BTO}+\mathrm{LCvol} \%$ were then prepared. However, the NPs form large aggregates directly after evaporating the solvents. Therefore, the first method of direct addition of NPs to the LC was preferably used.

Nematic samples cells are prepared with $1.5 \mathrm{~cm} \times 2 \mathrm{~cm}$ sized substrates of indium tin oxide (ITO) coated glasses (VisionTek Systems Ltd.) with a resistance of $10 \Omega / \square$. The glasses were cleaned by different solvents in a sonication bath for $30 \mathrm{~min}$. The ITO layer of the longer edges of the substrates was removed; leaving the ITO of the central (active) area of width $0.5 \mathrm{~cm} \times 1.5 \mathrm{~cm}$. Polyimide Kapton tape was used as a protective layer for the central area prior to the immersion of the substrates in hydrochloric acid (30\%) for $7 \mathrm{~min}$ to dissolve the ITO. The glasses were again cleaned for the final time, placed in a plasma chamber for $5 \mathrm{~min}$ with oxygen circulation to remove organic residues and increase the wetting properties of the surface. As an alignment layer a solution of polyvinyl alcohol, PVA, in water $(0.5 \mathrm{mg} / \mathrm{ml})$ was spin coated onto the substrates and dried at $100^{\circ} \mathrm{C}$, followed by uni-directional and antiparallel rubbing with a velvet cloth to produce planar alignment. The quality of the alignment layers was confirmed by optical polarising images of the liquid crystal. Without plasma cleaning, the spin coating of PVA did not produce satisfactory homogenous alignment as seen in Figure 1(a). The wetting properties were increased as the organic residues were removed in the plasma chamber, Figure 1(b). The ITO

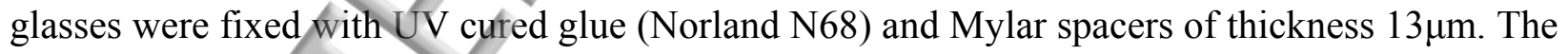
cell preparation procedure is schematically shown elsewhere. ${ }^{33}$ 


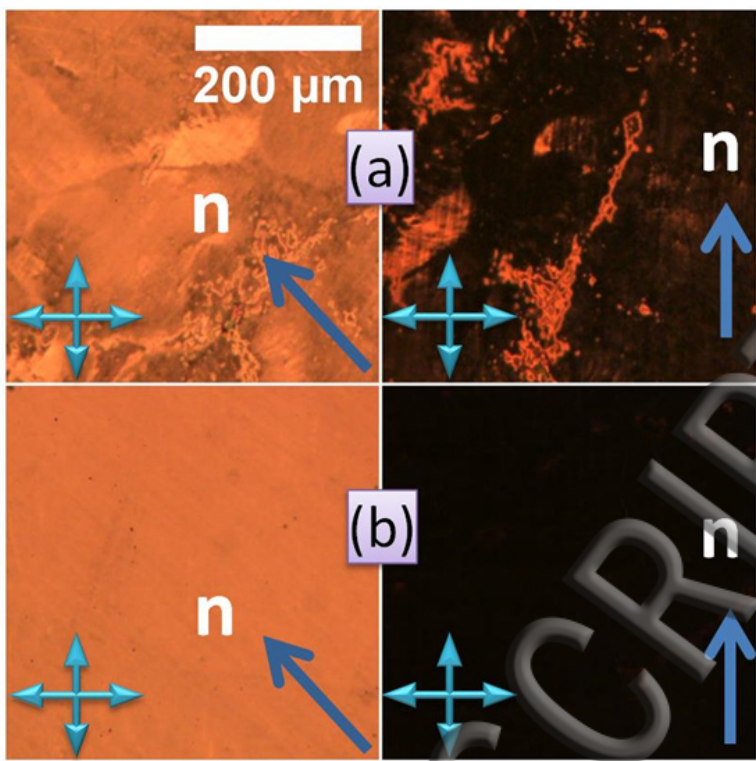

Fig. 1 Optical polarising images illustrating the quality of the alignment of cells filled with pure 5CB. The glass substrates are (a) only cleaned with solvents and detergents in the sonication bath and (b) treated additionally in a plasma chamber prior to spin coating PVA.

For ferroelectric dispersions BTO+FLC, commercial cells (AWAT, Poland), with a homogeneous alignment layer, cell thickness of $\mathrm{d}=5 \mu \mathrm{m}$, ITO area $\mathrm{A}=25 \mathrm{~mm} 2$ and ITO resistance $<10 \Omega / \square$ are used. In this case, the cells were filled by capillary action in the isotropic phase. The use of commercial cells was the only possibility to achieve the surface stabilised SSFLC geometry needed for electro-optic investigations.

\subsection{EXPERIMENTAL TECHNIQUE}

The size distributions of the $\mathrm{BaTiO}_{3}$ nanoparticles were determined by both scanning electron microscopy, SEM (FEI SIRION) and dynamic light scattering, DLS (Malvern). The nanoparticles are in form of a powder; with grains being aggregates of the actual nanoparticles. To produce samples suitable for SEM experiments, the nanopowder was suspended in isopropanol $(1 \mathrm{mg} / \mathrm{ml})$, and dispersed through the application of sonication, which resulted in aggregates being broken up into their constituent nanoparticles. Finally, a drop of this suspension was drop cast onto a conductive substrate of carbon films to leave homogeneously distributed nanoparticles on the substrate. 
Publishi.AgRenishaw RM1000 Raman spectrometer with 515.3nm laser wavelength (Spectra-Physics) and maximum output power of 50mW was employed for the Raman investigations on the BTO1 and BTO2 samples. Optical textures at increasing vol\% of nanoparticles doped into LCs were investigated using a Leica DMLP polarising microscope equipped with a digital image acquisition system (uEye CP). A precision temperature controller (Linkam TMS 94) of relative accuracy $\pm 0.1^{\circ} \mathrm{C}$ was used to control the sample temperature. Dielectric spectroscopic investigations were performed using an Agilent Precision LCR Meter E4980A, which was operated in the parallel equivalent circuit mode in a frequency range of $20 \mathrm{~Hz}$ to $2 \mathrm{MHz}$ with an applied measuring voltage of $\mathrm{Vac}=0.05-20 \mathrm{~V}$. The perpendicular component of the permittivity $\varepsilon^{\prime}{ }_{\perp}$ was measured below the Fréedericksz threshold $V_{\text {th }}$ while the parallel component of the permittivity $\varepsilon^{\prime}{ }_{\|}$was determined at electric fields well above the Fréedericksz transition.

The permittivity is determined from capacitance measurements by

$$
\varepsilon^{\prime}=\frac{C}{C_{0}}
$$

where $C_{o}$ is the capacitance of the empty cell and $C$ is the capacitance of the cell when filled with the liquid crystal-nanoparticle dispersion. It is further

$$
\tan \delta=\frac{\varepsilon^{\prime \prime}}{\varepsilon^{\prime}} \Rightarrow \varepsilon^{\prime \prime}=\varepsilon^{\prime} \cdot \tan \delta
$$

For the data analysis the generalised Havriliak-Negami equation was used to simultaneously fit the real and imaginary parts of the relaxation modes as a function of applied electric field frequency $\mathrm{f}^{36}$ :

$$
\varepsilon^{*}=\varepsilon_{\infty}^{\prime}+\frac{\Delta \varepsilon^{\prime}}{\left(1+\left(\mathrm{i} \frac{\mathrm{f}}{\mathrm{f}_{\mathrm{R}}}\right)^{\alpha}\right)^{\beta}}
$$


Publishiwgere $\varepsilon^{*}$ is the complex permittivity, $\varepsilon_{\infty}^{\prime}$ is the permittivity at high frequencies. $\Delta \varepsilon^{\prime}=\varepsilon_{\infty}^{\prime}$ $\varepsilon^{\prime}{ }_{0}$ is the dielectric strength, with $\varepsilon^{\prime}{ }_{0}$ the permittivity at low-frequency, $\mathrm{f}_{\mathrm{R}}$ is the relaxation frequency of a single mode, which is the Goldstone mode in the SmC* phase, $\alpha$ is a fit parameter which describes the width and height of the loss peak, and $\beta$ a further fit parameter which accounts for a possible asymmetry of the loss peak. In its simplest and symmetric case it is $\alpha=\beta=1$, relating to the Debye relaxation model. ${ }^{37}$ When $0 \leq \alpha \leq 1$ and $\beta=1$, the centre of the Cole-Cole plot semi-circle is shifted below the $\varepsilon^{\prime \prime}=0$ axis, referring to the Cole-Cole relaxation model. $^{38,39}$ Finally, when $\alpha=1$ and $0 \leq \beta \leq 1$, the relaxation peak shows an asymmetric distribution and a distorted semi-circle in the Cole-Cole plot, which is described by the ColeDavidson model. ${ }^{40,41}$ The most general case is described by the Havriliak-Negami model with $0 \leq \alpha \leq 1$ and $0 \leq \beta \leq 1$. The real and imaginary parts of the complex dielectric constant are related to each other by the Kramers-Kronig relation ${ }^{42}$ :

$$
\varepsilon^{*}=\varepsilon^{\prime}+\mathrm{i} \varepsilon^{\prime \prime}
$$

which implies that the frequency dependencies of $\varepsilon^{\prime}$ and $\varepsilon^{\prime \prime}$ have to be fitted simultaneously to obtain the dielectric strength $\Delta \varepsilon$ and the relaxation frequency $\mathrm{f}_{\mathrm{R}}$.

All of the electrooptic experiments were carried out using the same microscope based setup with an optical bandpass filter (Thorlab) of wavelength $632 \pm 10 \mathrm{~nm}$ (for the $5 \mathrm{CB}$ samples) and $544 \pm 10 \mathrm{~nm}$ (for the FLC) as a source of monochromatic light. For the threshold measurements, the voltage sweep of the LCR meter was synchronized with the transmitted light intensity recorded by a photodiode through a digital multimeter (Agilent 34401A). The electrooptic response time was measured with a carrier signal of $5 \mathrm{kHz}$ and $20 \mathrm{~V}$ amplitude, modulated with a $50 \mathrm{mHz}$ square wave, using a signal generator (Agilent 33220A). The transmitted light intensity was detected by a photodiode of rise time $100 \mu$ s and recorded on a digital oscilloscope (Tektronix TDS 2024C) after averaging 32 individual measurements to reduce statistical noise.

The electrooptic measurements relating to the $\mathrm{SmC}^{*}$ phase of the FLC were carried out on the same apparatus and included tilt angle, $\Theta$, spontaneous polarization, PS, and switching times, $\tau$ S. 
Publishiligst, to obtain a well aligned SmC* domain in the SSFLC geometry, an asymmetric electric field was applied to the sample while cooling from the isotropic phase. The tilt angle $\Theta$ was measured optically by applying a DC voltage of $10 \mathrm{~V}$ to the well aligned FLC texture to unwind the helix. When the sign of the applied electric field is reversed, the long axes of the FLC molecules are reoriented through twice the tilt angle (cone angle) achieving two bistable states. Under crossed polarisers, the transmitted light intensity I is given by ${ }^{1}$

$$
I=I_{0} \sin ^{2}(4 \Theta) \sin ^{2}\left(\frac{\Delta \mathrm{nd} \pi}{\lambda}\right)
$$

Where Io is the intensity of the incident light, $\Delta \mathrm{n}$ the birefringence, $\mathrm{d}$ the cell thickness, $\Theta$ the tilt angle, and $\lambda$ the wavelength of light. From the two dark positions for opposite bias field directions the cone angle $2 \Theta$ is determined to an accuracy of $\Theta$ within $\pm 1^{\circ}$. The spontaneous polarisation was measured by the current reversal method. ${ }^{43} \mathrm{~A}$ triangular electric field of amplitude $10 \mathrm{~V}$ and frequency $90 \mathrm{~Hz}$ was applied. The switching time $\tau_{\mathrm{s}}$ was measured by applying a square wave electric field of the same conditions.

\section{RESULT AND DISCUSSION}

\subsection{CHARACTERISATION OF THE PARTICLES}

Figure 2 (a) and (b) show typical scanning electron microscope (SEM) images of both sized nanoparticles, ВTO1 and BTO2, respectively. It is evident from the figures that the shape of the smaller size BTO1 particles is roughly spherical in contrast to the larger size BTO2 particles. The SEM images have been used to measure the size distributions depicted in Figure 2 (c). It is found that the mean diameter of BTO1 and BTO2 are approximately $80 \pm 20 \mathrm{~nm}$ and $240 \pm 80 \mathrm{~nm}$, respectively. These are somewhat larger than the values stated by the supplier, which are $\mathrm{BTO} 1=50 \mathrm{~nm}$ and $\mathrm{BTO} 2=200 \mathrm{~nm}$. 


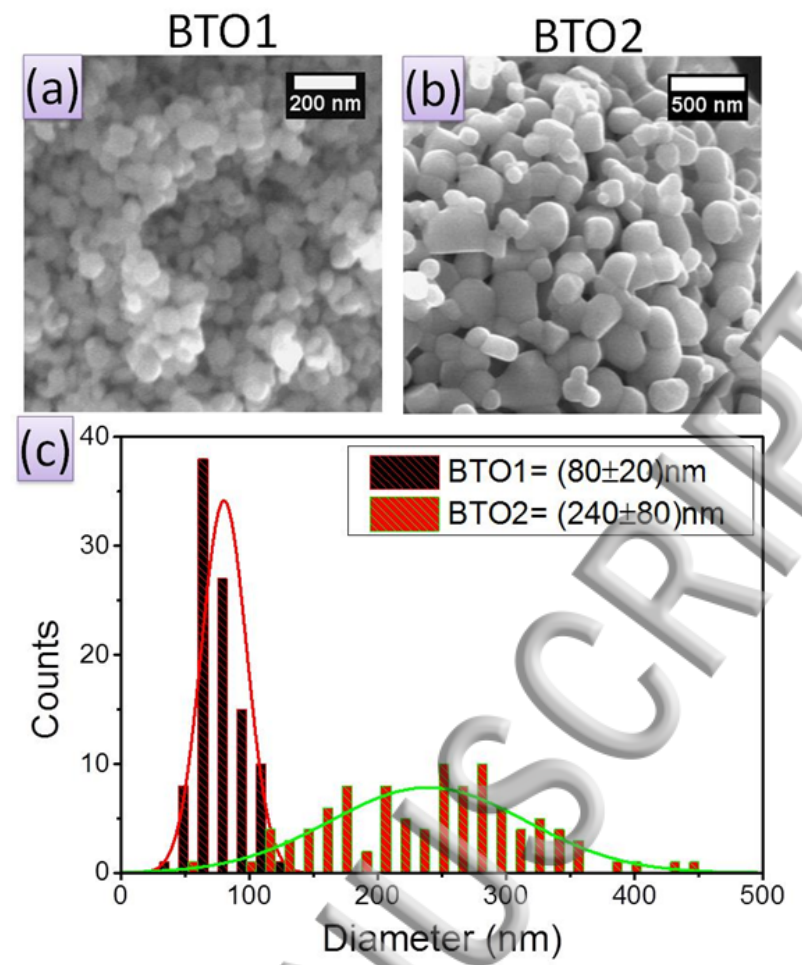

Fig. 2 SEM images of both sizes of $\mathrm{BaTiO}_{3}$ nanoparticles (a) BTO1 and (b) BTO2, and (c) their size distributions. Note the differences in scale for the SEM images.

To verify the sizes obtained, dynamic light scattering (DLS) was used to investigate the size distributions of the BTO nanoparticles in suspension. Various organic solvents such as dichloromethane, acetone, isopropanol, toluene, hexane, heptane, methanol, ethanol, and tetrahydrofuran were used as a medium to suspend the BTO nanoparticles. Isopropanol based suspensions were found to be the most stable, resulting in reproducible and good quality size distributions in the DLS experiment. The mean sizes that were measured by DLS were BTO1 $=78 \pm 28$ and BTO2 $=250 \pm 75$, equivalent to measurements from SEM.

Raman spectroscopy was used to examine ferroelectric properties of the nanoparticles as a function of particle size. Information about the shape of the unit cells of BTO1 and BTO2 were gained through Raman active vibration modes. ${ }^{44}$ It was found that for a tetragonal unit cell a ferroelectric crystal is observed, while a cubit unit cell obviously did not exhibit ferroelectricity. The Raman peaks for tetragonal BTO powder are centred at wavenumbers of $720 \mathrm{~cm}^{-1}, 515 \mathrm{~cm}^{-1}$, 
Publishi.38g $\mathrm{cm}^{-1}, 260 \mathrm{~cm}^{-1}$ and $185 \mathrm{~cm}^{-1} \cdot{ }^{45-47}$ It has previously been found that for a nanoparticle size range of 30-70nm, which are prepared by radio-frequency plasma chemical vapour deposition, the $\mathrm{BaTiO}_{3}$ nanoparticles are present in their pseudo-cubic phase. ${ }^{46}$ However, Millicent et. al have concluded that the ferroelectric tetragonal phase of $\mathrm{BaTiO}_{3}$, which are prepared chemically can be maintained down to $26 \mathrm{~nm}$ in size. ${ }^{47}$ This suggests that ferroelectricity depends on the methods of preparation of the nanoparticles.

The Raman shifts for different sizes of the commercial $\mathrm{BaTiO}_{3}$ nanoparticles that are employed in the present investigation are compared in Figure 3(a). The smaller size (BTO1) has been specified by the supplier as non-ferroelectric due to its size, while the larger size (BTO2) was provided as ferroelectric. It is evident from the figure that there are Raman peaks at wavenumbers of $720 \mathrm{~cm}^{-1}, 515 \mathrm{~cm}^{-1}, 305 \mathrm{~cm}^{-1}$ and $260 \mathrm{~cm}^{-1}$ for both NPs. However, the only difference lies in the amplitude of the peaks, as BTO2 exhibits stronger Raman peaks than BTO1, which was similarly reported by Millicent et. al. ${ }^{46,47}$ This indicats a strong decrease of ferroelectric tetragonal domains in the BTO1 batch. For a temperature dependent Raman spectroscopic investigation, Figure 3(b), the ferroelectric batch BTO2 was chosen. It is apparent from the figure that the peaks at $309 \mathrm{~cm}^{-1}$ and $720 \mathrm{~cm}^{-1}$ disappear between $150-200^{\circ} \mathrm{C}$, which was also observed by Klein et. al. ${ }^{4}$ These peaks were recognised as the tetragonal mode that disappears above the Curie-temperature, where the phase is entirely cubic. ${ }^{46}$ 
(a)

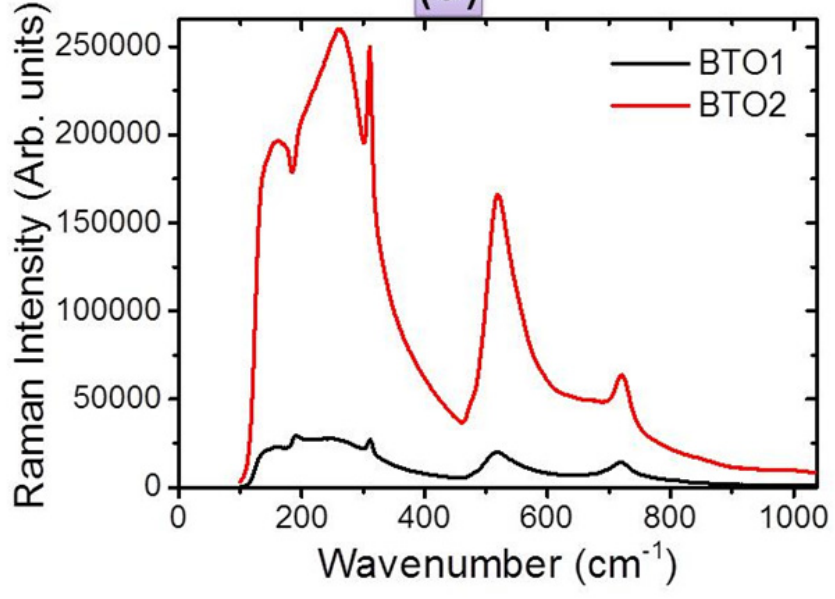

(b)

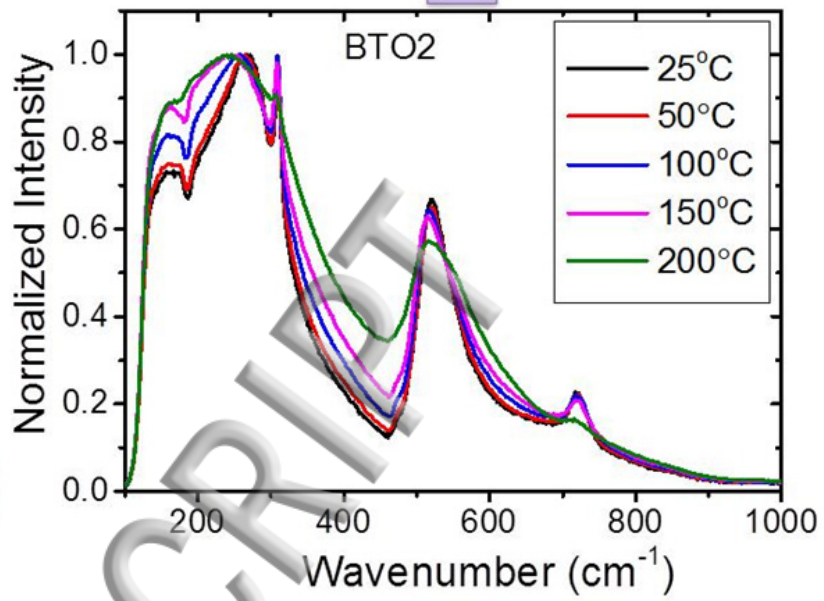

Fig. 3 Raman Spectrum of (a) BTO1 and BTO2 at room temperature, and (b) BTO2 Raman spectra at different temperatures.

\subsection{NANOPARTICLE DOPED NEMATIC LIQUID CRYSTAL (5CB)}

\subsubsection{OPTICAL POLARISING TEXTURES}

The suspension of $\mathrm{BaTiO}_{3}$ in a nematic liquid crystal medium can lead to various defects, particularly when the mixture is sandwiched between two plain glass slides with no alignment layer. For instance, at low concentrations of $0.5 \mathrm{vol} \%$ as the aggregates are below their percolation threshold, Schlieren textures are formed as shown in Figure 4(a). When the sample is viewed without polarisers, Figure 4(b), the individual micro-aggregates, were clearly visible (indicated by the circles). It is worthy to note that the particles are located in the singularity of the Schlieren defect, a behaviour which can be accounted for through the lowering of the defect elastic free energy. 
(a)

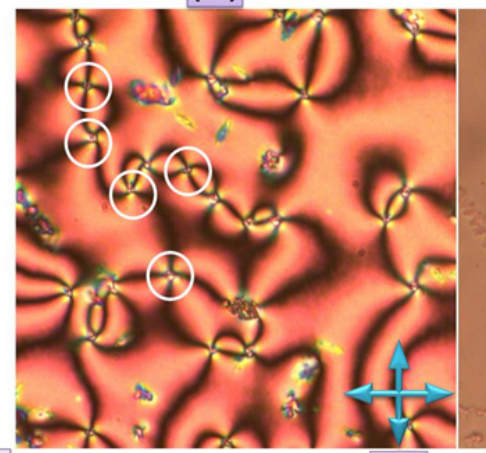

(d) (b)

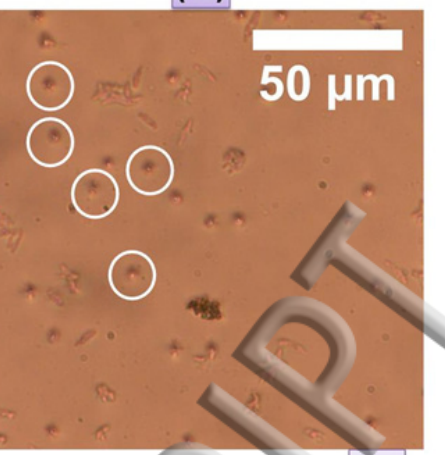

(e)

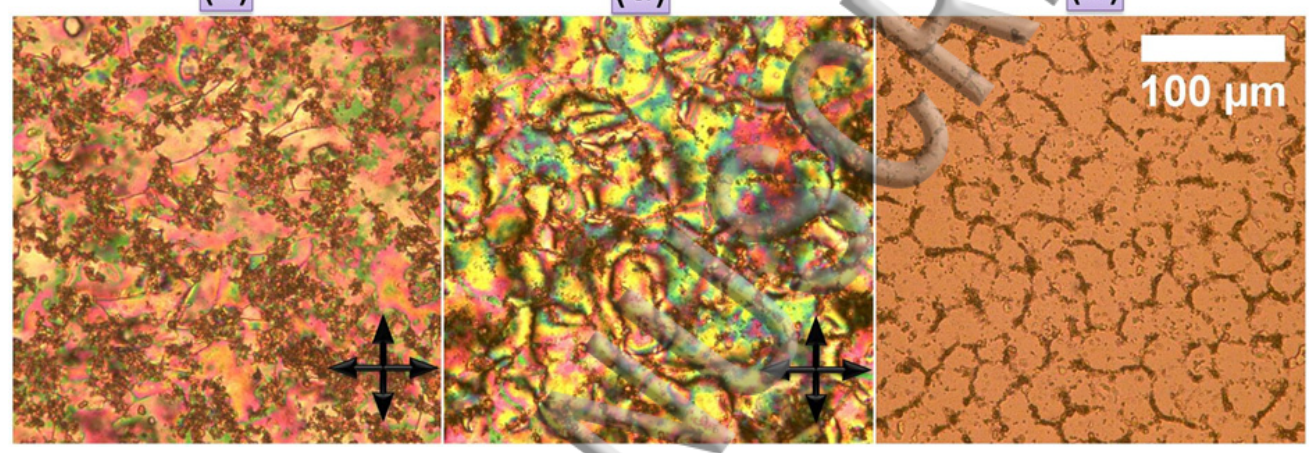

Fig. 4 Optical Polarizing photographs of BTO1+5CB sandwiched between two cover slip glasses: (a) A Schlieren texture is formed for a low particle concentration of $0.5 \mathrm{vol} \%$. (b) Corresponding photograph of (a) without polarisers, the particle aggregates being indicated by circles. Nanoparticle aggregates are localized in the singularity of the Schlieren defects to reduce the defect elastic free energy. (c) Nanoparticle aggregates for a concentration of $1 \mathrm{vol} \%$ before heating up to the isotropic phase, and (d) after the sample is cooled down $\left(1^{\circ} \mathrm{C} / \mathrm{min}\right)$ from the isotropic to the nematic phase. (e) The aggregates are ordered along the defect lines as depicted for the image of (d) without polarisers.

When the concentration of nanoparticles is increased, a larger number of aggregates is formed, and the Schlieren texture is disturbed. For BTO $+5 \mathrm{CB}$ at $1.0 \mathrm{vol} \%$ (Figure 4(c)) the NP aggregates are distributed randomly throughout the medium. As the sample is heated up to the isotropic temperature and cooled down to the nematic phase, Schlieren textures are observed where defects annihilate with time (Figure 4(d)). During the phase transition, the NPs assemble largely along the Schlieren brushes. As the defects annihilate, they leave a trace pattern of NP aggregates, following the defect centre path along the director field between a defect pair. A similar ordering of metallic nanoparticles has been observed in a dispersion with a cholesteric liquid crystal. ${ }^{48}$ However, in the aligned homogeneous planar cells no preferred direction for the ordering of aggregates in the nematic host was observed. Therefore, as shown in Figure 5, by 
This manuscript was accepted by J. Appl. Phys. Click here to see the version of record.

AlP

Publishilingreasing the concentration of BTO1 and BTO2, the agglomerates increased regardless of the size of the NPs.

(a)

(b)

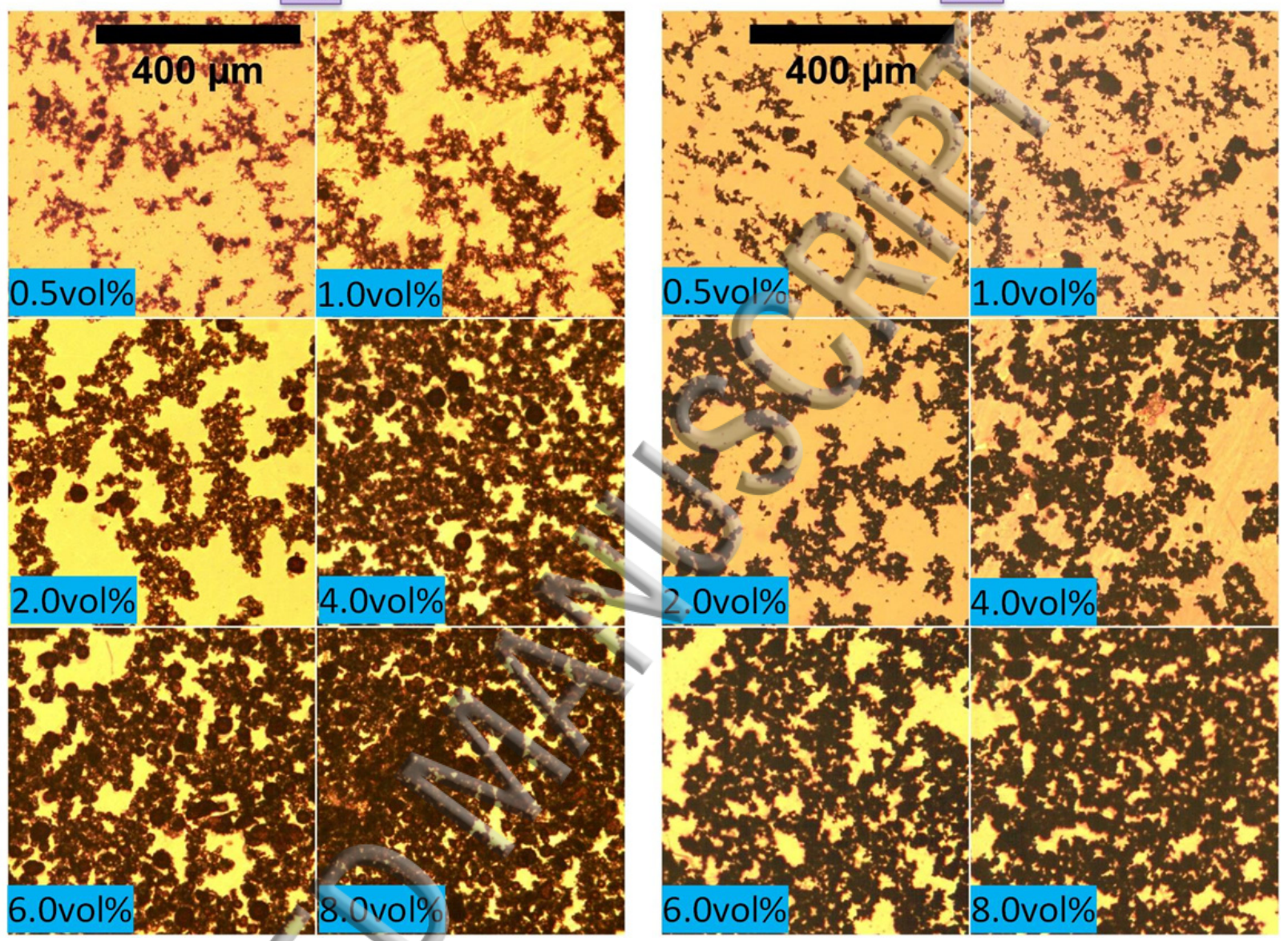

Fig. 5 Optical polarising images obtained from planar liquid crystal cells filled with BTO-5CB suspensions at concentrations of $0.5,1.0,1.5,2.0,4.0,6.0$ and $8.0 \mathrm{vol} \%$ as indicated in the texture photographs. Series (a) refers to small BTO1 and (b) to larger BTO2 NPs. The rubbing direction is at $45^{\circ}$ with the polarizers which are along the edges of the images.

Figure 5(a) and (b) show the optical polarising microscopic images of various concentrations of the suspensions $\mathrm{BTO} 1+5 \mathrm{CB}$ and $\mathrm{BTO} 2+5 \mathrm{CB}$, respectively. The agglomeration mechanism is similar for both paraelectric and ferroelectric NPs. Despite clear aggregation, the high loading concentrations were chosen, because at lower concentrations of $c<0.5 \mathrm{vol} \%$ the measured parameters were found to be in the range of the error of investigation. 
Publishiling:ddition, attempts were made to minimise the agglomeration of the NPs in the nematic medium, by coating the surface of the $\mathrm{BaTiO}_{3}$ nanoparticles with phosphoric acids, particularly octadecyl phosphonic acid (ODPA) (Sigma-Aldrich), as recommended by Paniagua et. al. ${ }^{49}$ Nevertheless, our investigations showed an even faster aggregation rate than that of the untreated nanoparticles, also with the use of an appropriate solvent, such/as dichloromethane and application of extensive sonication. Especially when the solvent/was evaporated, causing an isotropic to nematic transition, aggregation was enhanced strongly for the modified nanoparticles. For the investigations it was thus decided to employ nanoparticles without further surface treatment, dispersions being obtained solely by mixing and sonication.

\subsubsection{FRACTAL ANALYSIS OF THE BTO AGGREGATES}

Due to their obvious complexity the aggregates of $\mathrm{BaTiO}_{3}$ nanoparticles in the nematic medium 5CB suggest a further analysis by fractal geometric methods. The images with a resolution of $2048 \times 1088$ pixels were analysed by software Benoit 1.3 , using the box dimension method. The photographs of the textures were first thresholded and converted to binary images, a procedure which is exemplary depicted in Figure 6(a) and (b), respectively. The fractal dimension D is calculated from the proportionality

$$
N(l) \sim \frac{1}{l^{D}}
$$

where $N(l)$ is the number of boxes of length $l$ being occupied by the aggregate. 



Fig. 6 Exemplary demonstration of (a) image and (b) thresholded binary picture of the $\mathrm{BaTiO}_{3}$ aggregates (white). (c) Fractal dimension as a function of $\mathrm{BaTiO}_{3}$ vol\% concentration. The saturation dimension at high concentrations approaches $\mathrm{D}=1.9$. (Solid lines are a guide to the eye).

Figure 6 (c) shows the fractal dimension as a function of $\mathrm{BaTiO}_{3}$ concentration. It is found that the dimension saturates at a value of approximately $D=1.9$ at $4.0 \mathrm{vol} \%$ for both sizes of nanoparticles. It is noteworthy to mention that the saturation dimension of $D=1.9$ is equivalent to that of $2 \mathrm{D}$ percolation clusters at the percolation threshold. ${ }^{51}$ At this percolation threshold concentration of approximately $4.0 \mathrm{vol} \%$ the $\mathrm{BaTiO}_{3}$ aggregates form a single cluster that spans through the whole of the sample, which due its physical dimensions can be considered to be 2D. 


\section{PublishiB.8 3 NEMATIC-ISOTROPIC TRANSITION $\left(\mathbf{T}_{\mathrm{N}-1}\right)$}

The nematic to isotropic transition temperatures were examined using both polarising optical microscopy and differential scanning calorimetry DSC. Since a clear phase separation between liquid crystal and nanoparticles was seen, no significant change in $\mathrm{T}_{\mathrm{N}-\mathrm{I}}$ has been observed for any suspensions, neither with paraelectric or ferroelectric NPs, as expected. When examining the clearing temperature of a liquid crystal based dispersion, a single component LC such as 5CB is favoured over mixtures of compounds forming a liquid crystal. Using a solvent during sample preparation, the very low molecular weight components in a mixture could evaporate with the solvent. Li et al. have observed that $\mathrm{T}_{\mathrm{N}-\mathrm{I}}$ increased by $40^{\circ} \mathrm{C}$ for colloids of $\mathrm{BaTiO}_{3}$ and a nematic mixture, ${ }^{52}$ whereas in the current study the clearing point is not affected appreciably for $5 \mathrm{CB}$.

\subsubsection{DIELECTRIC PROPERTIES}

In Figure 7(a) and (b), the permittivity components $\varepsilon_{\perp}^{\prime}$ and $\varepsilon_{\|}^{\prime}$ are plotted as a function of $\mathrm{BaTiO}_{3}$ concentration. For the ferroelectric nanoparticle doped 5CB (BTO2+5CB) the parallel component of the dielectric constant $\varepsilon_{\|}^{\prime}$ increases stronger as a function of nanoparticle concentration than the perpendicular component $\varepsilon_{\perp}^{\prime}$. For the paraelectric nanoparticle (BTO1) suspensions $\varepsilon_{\|}^{\prime}$ is not significantly influenced. The changes are more obvious in the dielectric anisotropy $\Delta \varepsilon^{\prime}$ shown in Figure 7 (c). The observed behaviour could be attributed to the dipole orientation of the ferroelectric nanoparticles. For the low applied field regime below the threshold, resulting in the measurement of $\varepsilon_{\perp}^{\prime}$, the dipoles of the nanoparticles are randomly oriented and cancel overall. Measuring the component $\varepsilon_{\|}^{\prime}$, large fields are applied and the nanoparticle dipoles align to a certain extent, thus contributing to the dielectric constant. This is largely pronounced for the ferroelectric nanoparticles BTO2, while it is not observable for the paraelectric particles BTO1. Similarly, it has also been reported that $\Delta \varepsilon^{\prime}$ is found to increase with increasing concentration for other ferroelectric nanoparticles, for example tin hypothiodiphosphate $\mathrm{Sn}_{2} \mathrm{P}_{2} \mathrm{~S}_{6}{ }^{19}$ In addition, the enhanced dielectric anisotropies in the larger nanoparticle system $(\mathrm{BTO} 2+5 \mathrm{CB})$ indicates that the ferroelectricity of the NPs is maintained as the mixtures are prepared mechanically by using pastel and mortar. 
Publishingis worth noting that the changes in the measured dielectric permittivities were within experimental error when the loading concentrations were lower than $0.5 \mathrm{vol} \%$. However, such lower loading has been preferred in other investigations to maintain the director field least perturbed. $^{7,32,53}$

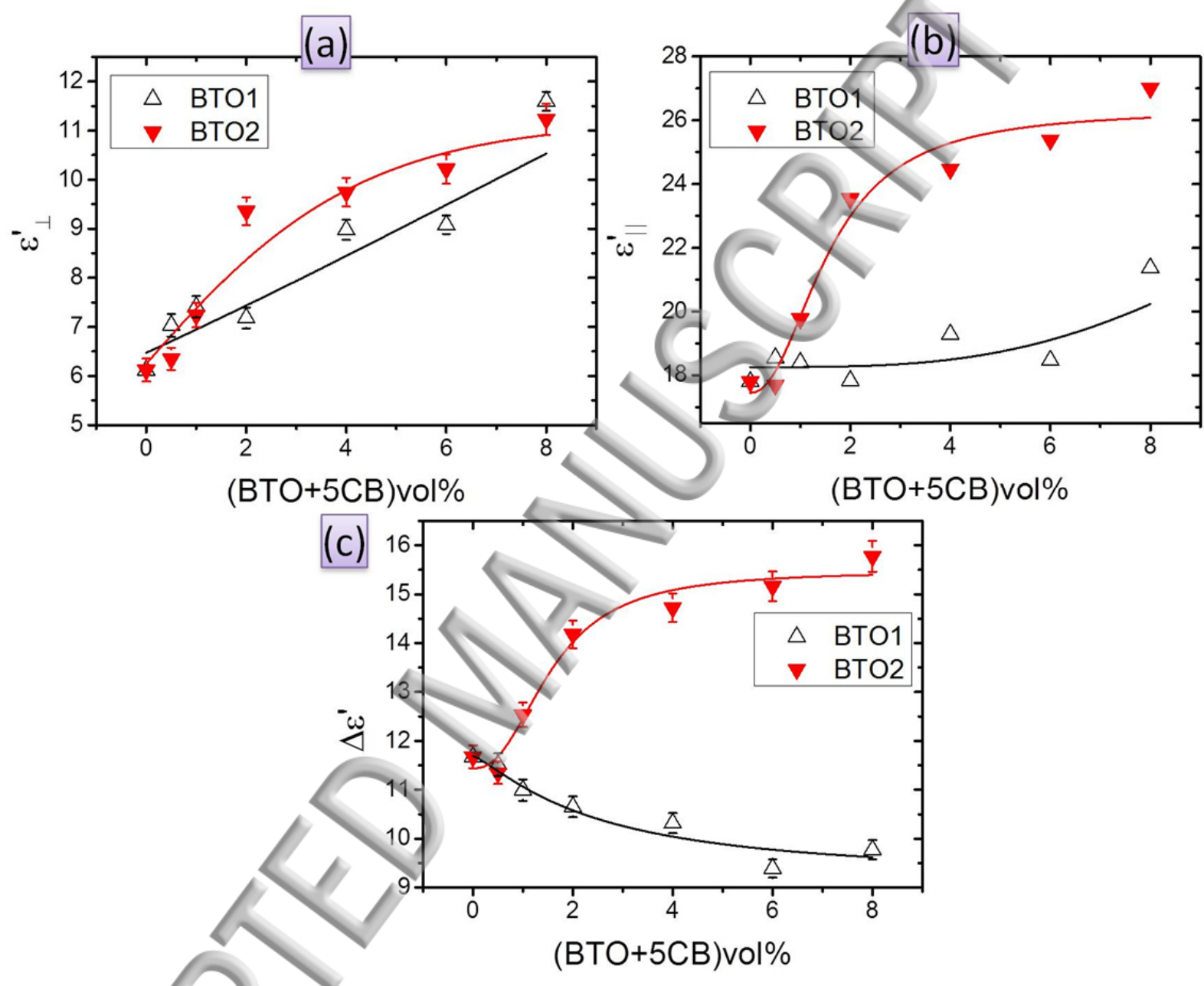

Fig. 7 Permittivity components (a) $\varepsilon^{\prime}{ }_{\perp}$ and (b) $\varepsilon^{\prime}{ }_{\|}$as well as (c) dielectric anisotropy $\Delta \varepsilon^{\prime}$ as a function of nanoparticle concentration for smaller, paraelectric BTO1 and larger, ferroelectric BTO2 nanoparticles in mixtures of $\mathrm{BaTiO}_{3}$ and $5 \mathrm{CB}$ for planar cells at $25^{\circ} \mathrm{C}$, and frequency $5 \mathrm{kHz}$. (Solid lines are a guide to the eye).

The effective dielectric anisotropy $\Delta \varepsilon_{\text {Susp. }}^{\prime}$ of the suspension can be defined as ${ }^{6}$ :

$$
\Delta \varepsilon_{S u s p .}^{\prime}=V_{L C} \Delta \varepsilon_{L C}^{\prime}+V_{N P} \Delta \varepsilon_{N P}^{\prime}
$$


Publishiwgere $V_{L C}$ and $V_{N P}$ are the volume fractions of the LC and the NPs respectively. Since the dielectric anisotropy of the ferroelectric NPs is approximately $2750,{ }^{11}$ and for pure $5 \mathrm{CB}$ about 11.5 , it is expected that $\Delta \varepsilon_{\text {Susp }}^{\prime}$. would be of the order 40 for $1.0 \mathrm{vol} \%$ of ferroelectric nanoparticles. Figure $7(\mathrm{c})$ shows that the measured $\Delta \varepsilon_{\text {Susp }}^{\prime}$ is considerably smaller than the one calculated. This could be explained by the fact that the local electric field is inhomogeneous throughout the medium due to the field depolarisation. ${ }^{20}$ In addition, Reshetnyak et al. have theoretically predicted that the effective dielectric anisotropy of a doped nematic liquid crystal depends on the spontaneous polarisation $P_{S}$ of the NPs as ${ }^{54}$

$$
\Delta \varepsilon_{S u s p .}^{\prime} \approx \Delta \varepsilon_{L C}^{\prime}+\frac{V_{N P} P s^{2} v F_{l}^{2}}{k_{B} T \varepsilon_{o}}
$$

where $\mathrm{v}$ is the nanoparticle volume $\left(\mathrm{v}=\frac{4}{3} \pi r^{3}\right)$, with $r$ the mean radius of NPs that is accessible through dynamic light scattering and SEM, $F_{l}$ the local field correction factor, $k_{B}$ the Boltzmann constant and $T$ the absolute temperature. Knowing the following parameters: nanoparticle volume $\approx 7.2 \times 10^{-21} \mathrm{~m}^{3}, \Delta \varepsilon_{L C}^{\prime} \approx 11.5, \Delta \varepsilon_{\text {Susp. }}^{\prime} \approx 16, V_{N P} \approx 0.08$, and supposing $F_{l} \approx 1$, the spontaneous polarization will be $\approx 3.2 \times 10^{-5} \mathrm{C} / \mathrm{m}^{2}$. This is much smaller than the bulk polarization $P_{S}$ of $\mathrm{BaTiO}_{3}$, which is of the order $0.26 \mathrm{C} / \mathrm{m}^{2}{ }^{55}$ It indicates that the polarization directions of the individual nanoparticles is only insignificantly oriented and ordered by an applied electric field.

\subsubsection{FRÉEDERICKSZ TRANSITION}

The threshold voltage corresponding to the Fréedericksz transition is given as ${ }^{56}$

$$
V_{t h}=\pi \sqrt{\frac{k_{11}}{\varepsilon_{o} \Delta \varepsilon^{\prime}}}
$$

Thus $\mathrm{V}_{\text {th }}$ depends on the competition between splay elastic constant $\mathrm{K}_{11}$ and the dielectric anisotropy $\Delta \varepsilon^{\prime}$. As indicated in Figure 8(a) the threshold voltage increases linearly with the 
Publishing increase in threshold voltage is independent on nanoparticle size. The elastic constant also increases with increasing nanoparticle concentration. This increase is slightly larger for the larger sized BTO2 than then smaller BTO1 particles (Figure 8(b)).
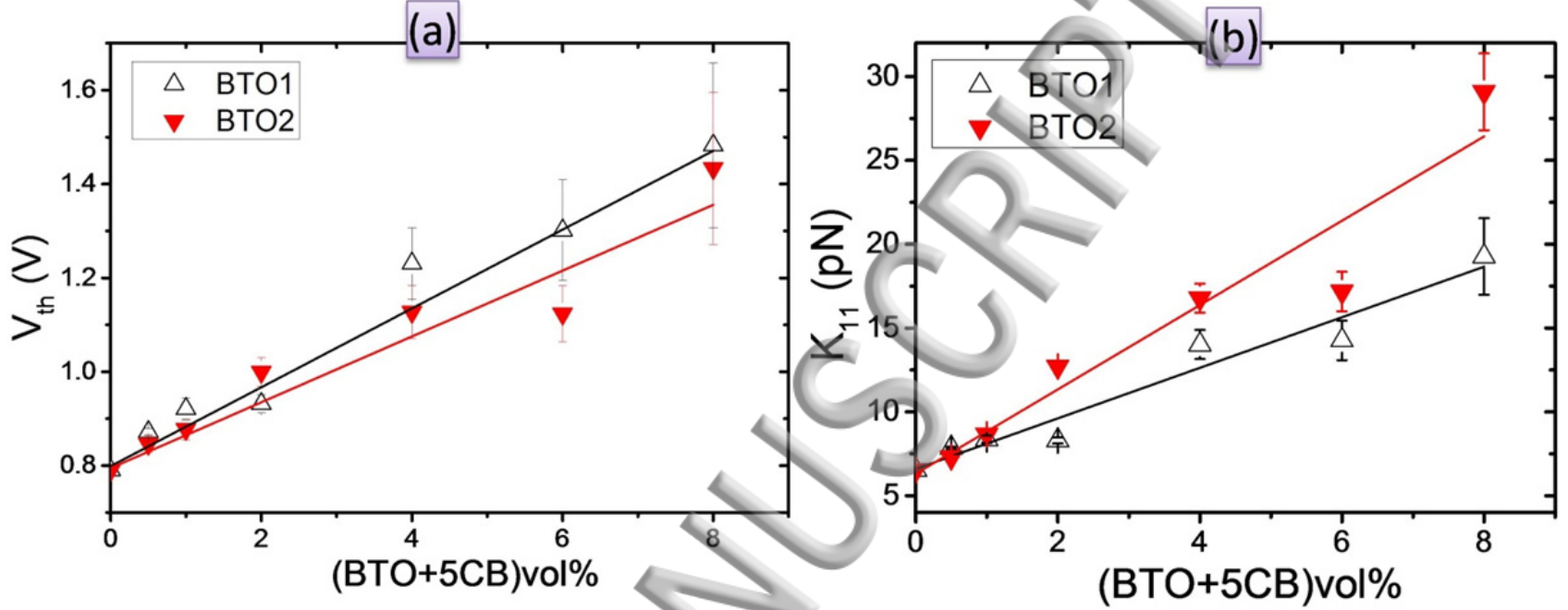

Fig. 8(a) Threshold voltage $\mathrm{V}_{\text {th }}$ and (b) splay elastic constant $\mathrm{K}_{11}$ as a function of nanoparticle concentration. (Solid lines are a guide to the eye).

\section{ELECTRO-OPTIC PROPERTIES}

The time required for the director to respond to an applied voltage above $\mathrm{V}_{\text {th }}, \tau_{\text {rise }}$, and time to return to its zero volt state, $\tau_{\text {decay }}$, are given as ${ }^{56,57}$

$$
\begin{gathered}
\tau_{\text {rise }}=\frac{\gamma_{1} d^{2}}{\Delta \varepsilon^{\prime} \varepsilon_{o} V^{2}-\pi^{2} K_{11}} \\
\tau_{\text {decay }}=\frac{\gamma_{1} d^{2}}{\pi^{2} K_{11}}
\end{gathered}
$$

where $\gamma_{1}$ is the rotational viscosity, $d$ the cell gap, $\Delta \varepsilon^{\prime}$ the dielectric anisotropy, $K_{11}$ the splay elastic constant, and $\mathrm{V}$ the applied voltage. Figure 9 depicts both times as a function of $\mathrm{BaTiO}_{3}$ particle concentration for both the smaller and the larger NPs. The electric field driven switching 
Publishiprggcess, or on-time, is found to be practically independent of size and concentration, within the limits of error (Figure 9(a)). On the contrary the off-time for the elastically driven reorientation is observed to decrease with increasing nanoparticle concentration as depicted in Figure 9(b). This implies that the elastic interactions between liquid crystal and nanoparticles increases with increasing concentration, which was already reflected by the increase of the elastic splay constant $\mathrm{K}_{11}$ (Figure $8(\mathrm{~b})$ ). It is interesting to note that this behaviour is also practically independent of nanoparticle size.
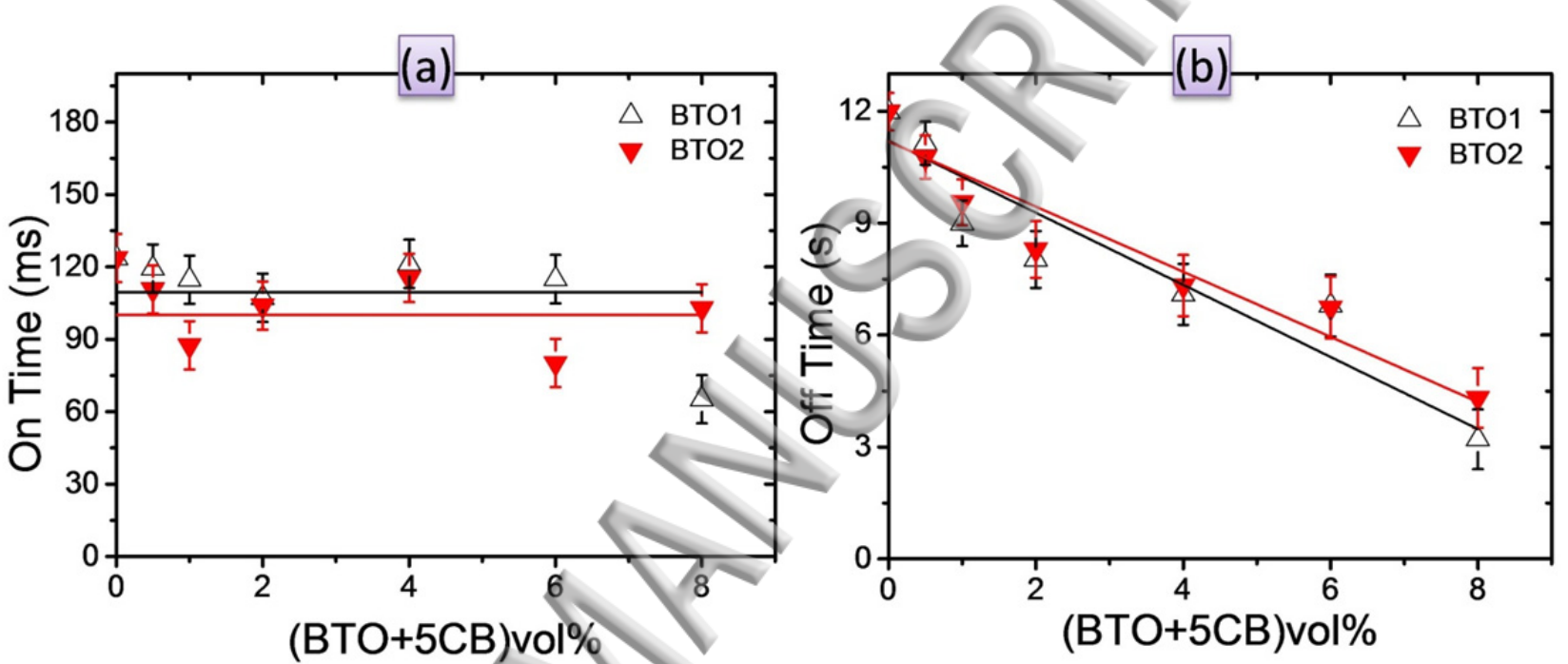

Fig. 9 Electrooptic response times as a function of $\mathrm{BaTiO}_{3}$ concentration in 5CB. (a) On-time for the electric field driven process and (b) off-time for the elastically driven reorientation process after field removal. (Solid lines are a guide to the eye).

\subsection{NP DOPED FERROELECTRIC LIQUID CRYSTAL FLC}

\subsubsection{OPTICALPOLARISING TEXTURES}

The optical polarising microscopic images of various concentrations of BTO doped FLC are shown in Figure 10. It can be seen that the texture of the suspensions appears similar to that of the pure FLC, Figure 10(a). This means that the NPs are well dispersed without significant aggregates and not perturbing the director field. In contrast to the nematic liquid crystal of a single molecular species $5 \mathrm{CB}$, the particle dispersion for the commercial multi-component FLC is observed to be enhanced. In the latter case we do not observe large aggregates of nanoparticles 
Publishibg well dispersed particles, except for regions of domain boundaries. This implies that the dispersion stability is strongly dependent on the nature of the liquid crystal host.

Since PVA spin coating did not provided sufficient quality of alignment for the FLC material and its suspensions, commercial cells with a cell gap of $\mathrm{d} \approx 5 \mu \mathrm{m}$ are used. Thus, any aggregates larger than the cell gap would be size excluded during the cell filling process in the isotropic phase.

Fig. 10 Optical polarising images of pure FLC (a), (BTO1+FLC)1.0vol\% (b), (BTO1+FLC)2.0vol\% (c), (BTO2+FLC)1.0vol\% (d), (BTO2+FLC)2.0vol\% (e) at room temperature. The sandwich cell gap is approximately $\mathrm{d}=5.0 \mu \mathrm{m}$. 
Publishibgon cooling from the isotropic phase, (similar to the $\mathrm{BTO}+5 \mathrm{CB}$ dispersions) the nanoparticles are aggregating at the defects, here the fan-shaped domain boundaries, of the $\mathrm{SmC}^{*}$ phase. This behaviour is demonstrated in Figure 11(a) and (b), showning the liquid crystal between crossed polarisers and without polarizers, respectively.

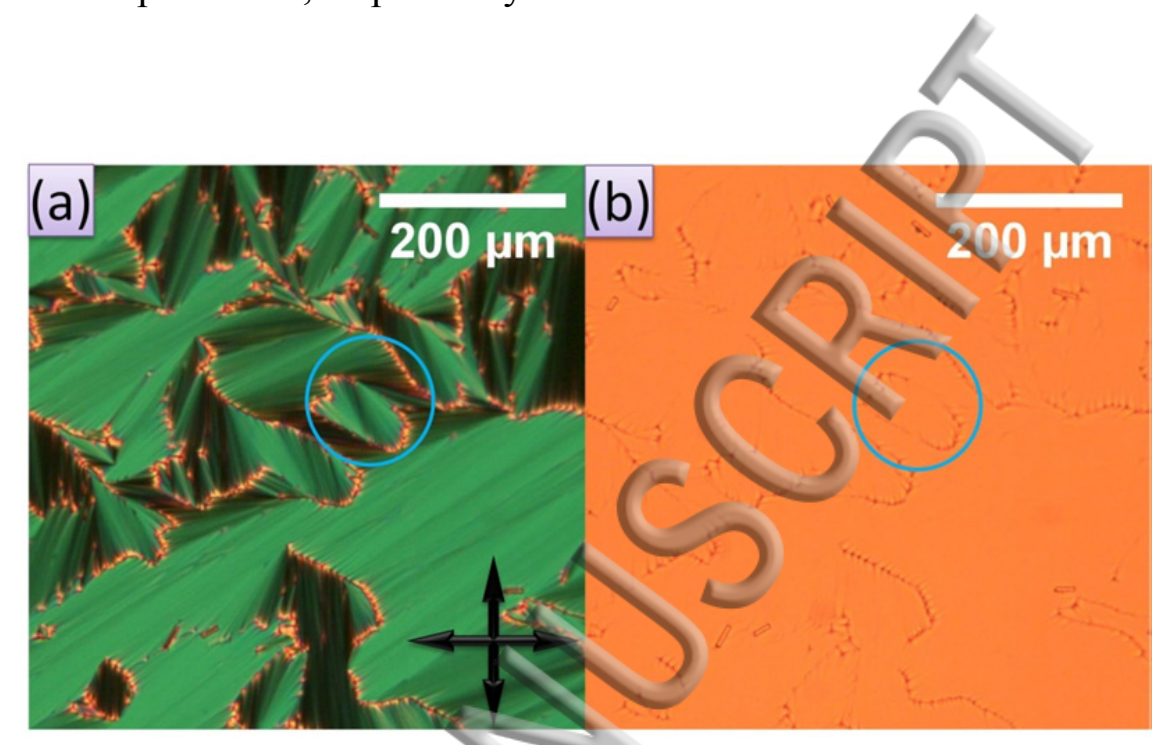

Fig. 11 Photographs of (BTO2+FLC)2.0vol\% with (a) crossed polarisers and (b) without polarisers. The nanoparticles are aggregating in the domain boundaries of the $\mathrm{SmC}^{*}$ phase.

\subsubsection{TILT ANGLE, SPONTANEOUS POLARISATION, SWITCHING TIME}

The temperature dependence of the most important FLC quantities for applications, tilt angle $\Theta$, switching time $\tau_{S}$ and spontaneous polarisation $P_{S}$ for the pure FLC and doped samples as a function of reduced temperature $T-T_{c}$, with $T_{C}$ being the transition temperature from SmA* to SmC*, are shown in Figure 12(a), (b) and (d), respectively. The spontaneous polarization was calculated from the area of the current reversal peaks $A_{s}$, using equation (12). The evolution of the polarization reyersal current at changing temperatures is exemplary shown in part (c) of Figure 12.

$$
P_{S}=\frac{A_{s}}{2 R A}=\frac{1}{2 R A} \int \Delta V d t
$$


Publishiwgere $R$ is the resistance of the current-to-voltage amplifier and $A$ is the active electrode area of the sandwich cell.
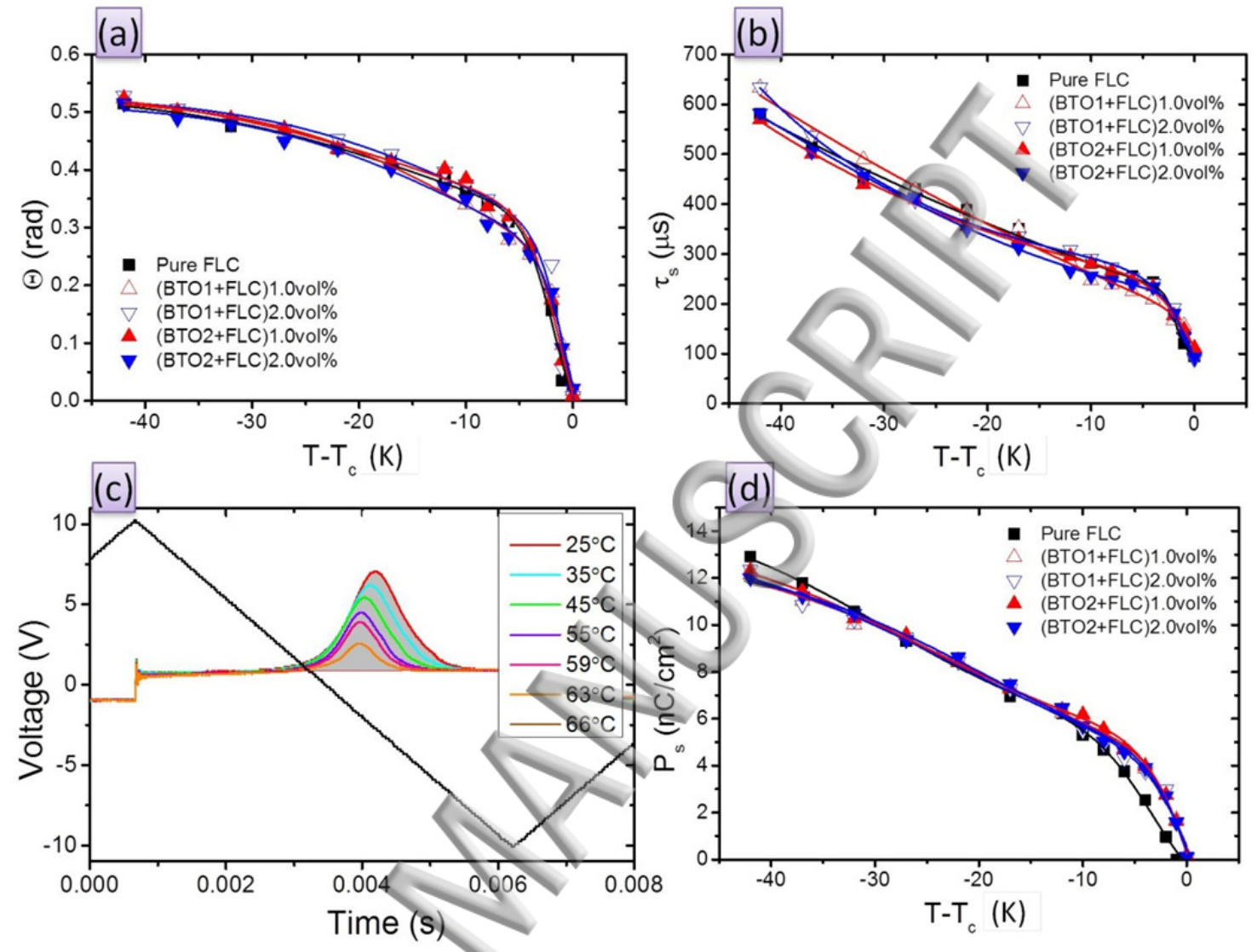

Fig. 12 Variation of (a) tilt angle $\Theta$, (b) switching time $\tau_{s}$ and (d) spontaneous polarisation $P_{s}$ with reduced temperature T- $\mathrm{T}_{\mathrm{c}}$ for the pure FLC and doped samples. Part (c) demonstrates the temperature evolution of the polarization reversal current for an applied triangular electric field of amplitude $\mathrm{E}=2 \mathrm{~V} / \mu \mathrm{m}$. Solid lines are a guide to the eye.

As the material is heated in the $\mathrm{SmC}^{*}$ phase, the tilt angle and corresponding spontaneous polarisation decreases as expected, with no significant differences between pure and doped samples, or smaller (BTO1, paraelectric) and larger (BTO2, ferroelectric) nanoparticles. At the Curie temperature of $\mathrm{T}_{\mathrm{c}}=66^{\circ} \mathrm{C}$ the ferroelectric behaviour of the liquid crystal and the dispersions vanishes completely. It has been shown that an increase of spontaneous polarisations of ferroelectric nanoparticle doped FLCs is much lower than if it is calculated from the following equation. 


$$
P_{S, \text { susp. }}=V_{L C} P_{S, L C}+V_{N P} P_{S, N P}
$$

The main reasons behind this discrepancy could be attributed to the random distribution of the ferroelectric NPs in the FLC host. Thus the corresponding dipole moments will largely cancel in fact reducing the total spontaneous polarisation $P_{S}$ due to dilution of the dipole density. Similarly, the tilt angle and response times are little influenced by suspending ferroelectric NPs in the ferroelectric liquid crystal host. The general results of experimentally determined parameters $\Theta$ and $P_{S}$ for the here studied FLC Felix M4851/050 are thus in accordance with previous studies. ${ }^{59,60}$

\subsubsection{DIELECTRIC SPECTROSCOPY}

Dielectric spectroscopy does not only reveal the dielectric constants and their anisotropy, but also provides information about potential relaxation processes. In the $\mathrm{SmC}^{*}$ phase these are namely the Goldstone mode, related to director fluctuation on the tilt cone, and the Soft mode, which is related to fluctuations in the modulus of the tilt angle. While the former is usually observed without hesitation, the latter relaxation mode can be quite small and is often only observable in the close vicinity of the $\mathrm{SmA}^{*}$-SmC* (paraelectric to ferroelectric) phase transition. Dielectric spectroscopy can further be employed to estimate the temperature dependence of the rotational viscosity, as shown below. The temperature dependence of the dielectric properties for the pure and nanoparticle doped FLC is shown in Figure 13. For all five samples, the effect of different concentrations of both NPs (small size, paraelectric BTO1, open symbols) and (larger size, ferroelectric BTO2, closed symbols) on the pure FLC are depicted for the permittivity $\varepsilon^{\prime}$, the dielectric loss $\varepsilon^{\prime \prime}$ and the conductivity $\sigma$. 

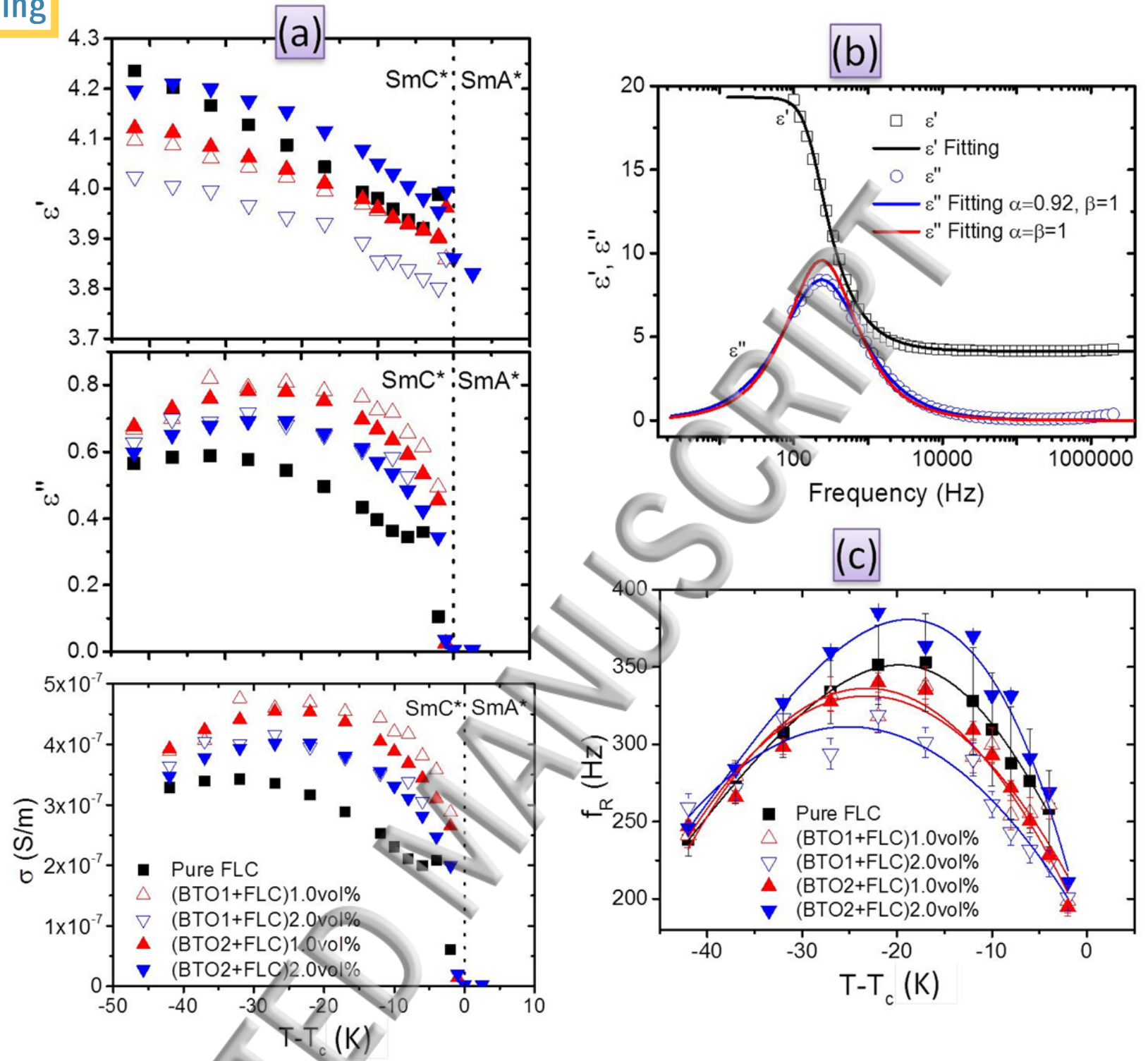

Fig. 13 Reduced temperature dependence of (a) the permittivity $\varepsilon^{\prime}$ (top), dielectric loss $\varepsilon^{\prime \prime}$ (middle) and electrical conductivity $\sigma$ (buttom) at 10kHz. (b) depicts an example for the simultaneous fitting procedure according to the Harvilliak-Negami equation, as employed to obtain the (c) Goldstone mode relaxation frequencies $f_{R}$ as a function of reduced temperature T- $\mathrm{T}_{\mathrm{c}}$. Solid lines are a guide to the eye.

The Goldstone mode can be determined by the generalised Havriliak-Negami equation (3). An example for a fit of experimental data is shown in Figure 13(b) in comparison to both the Debye model with $\alpha=\beta=1$, and the Cole-Cole model with $0 \leq \alpha \leq 1$ and $\beta=1$. It is evident from the figure that the experimental data of the Goldstone mode relaxation is well fitted with the 
Publishiđgle-Cole model, which is in accordance to other SmC* materials. ${ }^{61-63}$ The permittivity $\varepsilon^{\prime}$ is only little affected by the dispersed nanoparticles, changing values within experimental error of approximately 0.1 . The dielectric loss $\varepsilon "$ values appear to slightly increase for the nanoparticle doped systems, as compared to the neat FLC mixture. Small concentrations of nanoparticles seem to have a larger effect than more excessive doping, a behaviour which is also observed for the electric conductivity $\sigma$.

The temperature dependence of the Goldstone mode relaxation frequency for the pure FLC and doped samples is shown in Figure 13(c). The trend indicates that relaxation frequencies $f_{R}$ increase with temperature, reach a maximum at approximately $20 \mathrm{~K}$ below the Curie temperature, and then decrease again when approaching further to the $\mathrm{SmC}^{*}$ to $\mathrm{SmA}^{*}$ phase transition. A similar behaviour has been reported for Goldstone mode-relaxation frequencies before. ${ }^{62,64,65} \mathrm{It}$ might be caused by the competition between the temperature dependence of the elastic constant and that of the viscosity in the $\mathrm{SmC}^{*}$ phase (equation (14)) as given by ${ }^{66}$

$$
f_{R} \sim \frac{k_{e f f}}{\eta}
$$

where $k_{\text {eff }}$ is an effective elastic constant and $\eta$ the viscosity for a director reorientation along the tilt cone.

\subsubsection{ROTATIONAL VISCOSITY}

As mentioned above, the rotational viscosity $\eta$ can be determined by several different methods, from electrooptic response times (equation $(16)$ ) ${ }^{67}$ the polarization reversal current (equation $(17))^{68}$ and dielectric spectroscopy (equation 18$)^{66,69}$ : 


$$
\begin{gathered}
\eta=\tau_{s} P_{s} E \\
\eta=\frac{A \tau_{s} P_{s}^{2} E_{m}}{I_{s}^{m} \sin ^{2} \Theta}
\end{gathered}
$$

where $E_{m}$ is the electric field at the maximum value of electric current $I_{s}^{m}$, and

$$
\eta=\frac{1}{4 \pi \varepsilon_{o}} \frac{1}{\Delta \varepsilon_{G} f_{R}}\left(\frac{P_{s}}{\Theta}\right)^{2}
$$

where $\varepsilon_{o}$ the permittivity of free space, and $\Delta \varepsilon_{G}$ is the dielectric strength of the Goldstone mode. The experimental results of $\eta\left(T-T_{C}\right)$ for pure and doped samples are shown in Figure 14 for all three outlined methods. In all cases similar values are obtained for the rotational viscosity, which decreases with increasing temperature, as expected. A more rapid decrease is observed in the vicinity of the $\mathrm{SmC}^{*}$ to $\mathrm{SmA}^{*}$ transition, which can be attributed to the softening of the potential and the electroclinic effect. No clear trends or significant differences in values between the neat ferroelectric liquid crystal and the nanoparticle doped samples are observed. 


\section{Publishing}

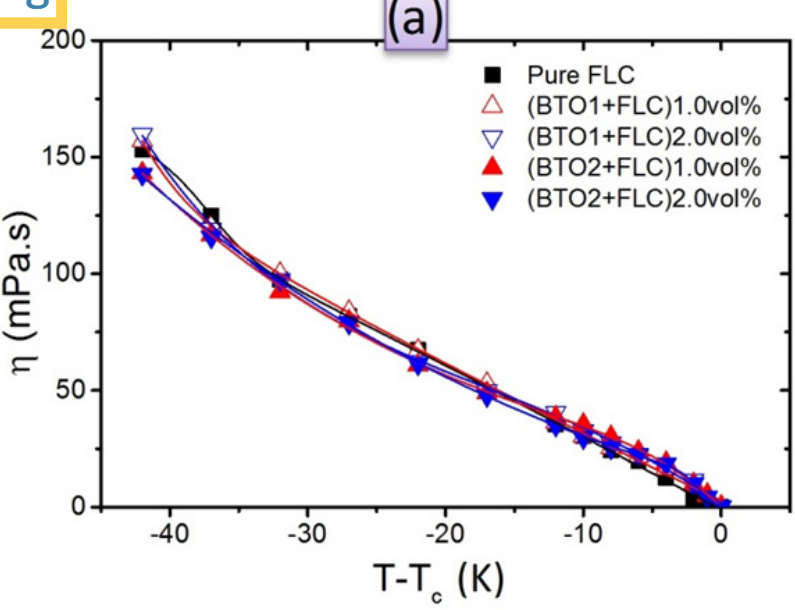

(a)

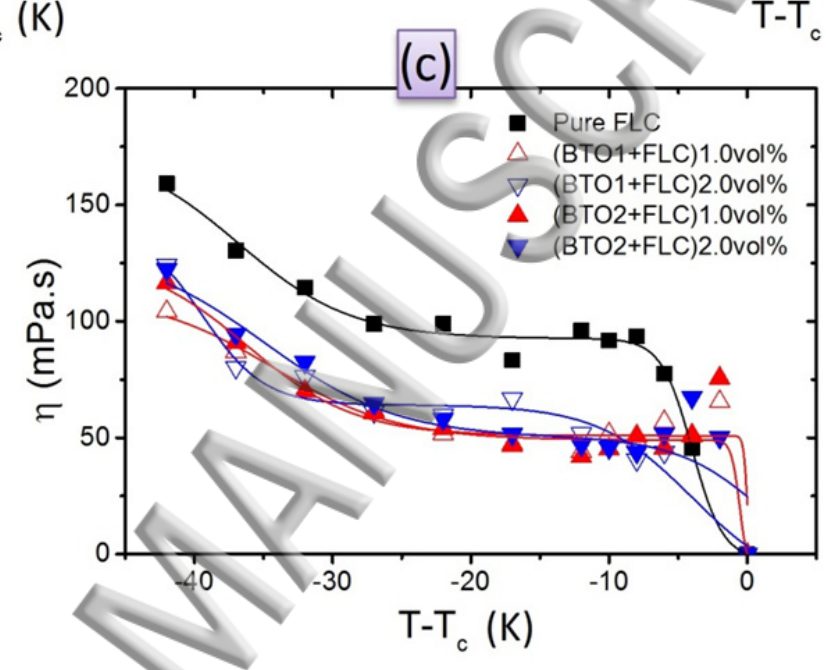

(b)

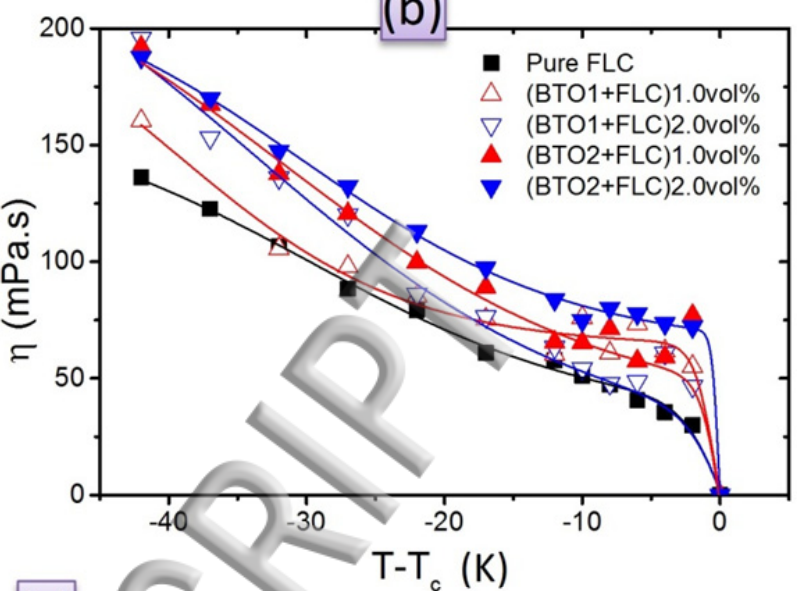

Fig. 14 Temperature dependence of the rotational viscosity $\eta$ determined from (a) electro-optic response times (eq. (15)), (b) polarization reversal current (eq.(16)) and (c) dielectric spectroscopy (eq. (17)) for the pure FLC and the nanoparticle doped systems. Solid lines are a guide to the eye. 


\section{CONCLUSION}

Two different sizes of $\mathrm{BaTiO}_{3}$ nanoparticles were doped in the nematic phase $(5 \mathrm{CB})$ and the ferroelectric SmC* phase (Felix M4851/050) of a liquid crystal. The size ranges of the NPs were carefully chosen to compare the effects of size-dependent-ferroelectricity on the electrooptic and dielectric properties of the liquid crystal - nanoparticle dispersions. It was found that for very low concentrations smaller than approximately $0.5 \mathrm{vol} \%$ the nanoparticles did not influence the liquid crystal properties at all. At larger particle concentrations the threshold voltage and the elastic constant of the nematic LC increased for the addition of both paraelectric as well as ferroelectric nanoparticles, while the dielectric anisotropy strongly increased for the ferroelectric dopant, in contrast to the constant values observed for the paraelectric dopant particles. The observed behaviour is consistently reflected in the electrooptic performance of the dispersions, where the response times of the electric field driven switching-on process remain constant, while the elastically driven field-off response is enhanced. In contrast to the observed changes in physical parameters and electro-optic performance of the nematic liquid crystal, neither of the nanoparticles had any significant effects on the properties and performance of the ferroelectric liquid crystal. For the latter dispersions, independent of size and properties of the nanoparticles, practically no significant effect on tilt angle, spontaneous polarization, response time, viscosity or dielectric relaxation of the FLC - nanoparticle dispersions was observed.

\section{Acknowledgements}

S. A-Z. would like to thank the KRG-Iraqi government for a HCDP scholarship. Help with the Raman experiments by Helen Gleeson is gratefully acknowledged. 


\section{PublishingREFERENCES}

${ }^{1}$ I. Dierking, Textures of Liquid Crystals (Wiley-VCH Verlag GmbH \& Co. KGaA, Weinheim, FRG, 2003).

2 P.J. Collings and M. Hird, Introduction to Liquid Crystals Chemistry and Physics (Taylor \& Francis, London; Bristol, PA, 1997).

${ }^{3}$ P.G. De Gennes and J. Prost, The Physics of Liquid Crystals, 2nd ed.(Oxford University Press, Oxford, New York, 1993).

${ }^{4}$ S. Klein, R.M. Richardson, R. Greasty, R. Jenkins, J. Stone, M.R. Thomas, and A. Sarua, Philos. Trans. R. Soc. A Math. Phys. Eng. Sci. 371, 20120253 (2013).

${ }^{5}$ F. Li, J. West, A. Glushchenko, C.I. Cheon, and Y. Reznikov, J. Soc. Inf. Disp. 14, 523 (2006).

${ }^{6}$ Y. Reznikov, O. Buchnev, O. Tereshchenko, V. Reshetnyak, A. Glushchenko, and J. West, Appl. Phys. Lett. 82, 1917 (2003).

${ }^{7}$ M. Gupta, I. Satpathy, A. Roy, and R. Pratibha, J. Colloid Interface Sci. 352, 292 (2010).

${ }^{8}$ J.-F. Blach, S. Saitzek, C. Legrand, L. Dupont, J.-F. Henninot, and M. Warenghem, J. Appl. Phys. 107, 74102 (2010).

${ }^{9}$ I. Dierking, G. Scalia, and P. Morales, J. Appl. Phys. 97, 44309 (2005).

${ }^{10}$ M.R. Herrington, O. Buchnev, M. Kaczmarek, and I. Nandhakumar, Mol. Cryst. Liq. Cryst. 527, 72/[228] (2010).

${ }^{11}$ A. Glushchenko, C. Il Cheon, J. West, F. Li, E. Büyüktanir, Y. Reznikov, and A. Buchnev, Mol. Cryst. Liq. Cryst. 453, 227 (2006).

${ }^{12}$ H.M. Atkuri, K. Zhang, and J.L. West, Mol. Cryst. Liq. Cryst. 508, 183 (2009).

${ }^{13}$ S. a. Basun, G. Cook, V.Y. Reshetnyak, a. V. Glushchenko, and D.R. Evans, Phys. Rev. B 84, 24105 (2011).

${ }^{14}$ H. Atkuri, G. Cook, D.R. Evans, C.-I. Cheon, A. Glushchenko, V. Reshetnyak, Y. Reznikov, J. West, and K. Zhang, J. Opt. A Pure Appl. Opt. 11, 24006 (2009).

15 O. Kurochkin, H. Atkuri, O. Buchnev, A. Glushchenko, O. Grabar, Karapinar R, V. Reshetnyak, J. West, and Y. Reznikov, Condens. Matter Phys. 13, 33701 (2010).

${ }^{16}$ Y.S.C. M. R. Hakobyan, R. B. Alaverdyan, R. S. Hakobyan, Armen. J. Phys. 7, 11 (2014).

${ }^{17}$ A. Mikułko, P. Arora, A. Glushchenko, A. Lapanik, and W. Haase, EPL (Europhysics Lett. 87, 27009 (2009).

18 N. Podoliak, O. Buchnev, M. Herrington, E. Mavrona, M. Kaczmarek, A.G. Kanaras, E. Stratakis, J.-F. Blach, J.-F. Henninot, and M. Warenghem, RSC Adv. 4, 46068 (2014).

19 Y. Reznikov, O. Buchnev, A. Glushchenko, V. Reshetnyak, O. Tereshchenko, and J. West, in Emerg. Liq. Cryst. Technol, edited by L.-C. Chien (2005), p. 171.

${ }^{20}$ A. Rudzki, D.R. Evans, G. Cook, and W. Haase, Appl. Opt. 52, E6 (2013). 
Publishîig. Wang, W. He, X. Xiao, M. Wang, M. Wang, P. Yang, Z. Zhou, H. Yang, H. Yu, and Y. Lu, J. Niater. Chem. 22, 19629 (2012).

${ }^{22}$ A. Lorenz, N. Zimmermann, S. Kumar, D.R. Evans, G. Cook, and H.-S. Kitzerow, Phys. Rev. E 86, 51704 (2012).

${ }^{23}$ Y. Reznikov, Liq. Cryst. Beyond Displays Chem. Physics, Appl. 403 (2012).

${ }^{24}$ I. Coondoo, P. Goel, A. Malik, and a. M. Biradar, Integr. Ferroelectr. 125, 81 (2011).

${ }^{25}$ O. Kurochkin, O. Buchnev, A. Iljin, S.K. Park, S.B. Kwon, O. Grabar, and Y. Reznikov, J. Opt. A Pure Appl. Opt. 11, 24003 (2009).

${ }^{26}$ Y. Lin, A. Daoudi, A. Segovia-Mera, F. Dubois, C. Legrand, and R. Douali, Phys. Rev. E 93, 62702 (2016).

27 M.F. Prodanov, O.G. Buluy, E. V. Popova, S.A. Gamzaeva, Y.O. Reznikov, and V. V. Vashchenko, Soft Matter 12, 6601 (2016).

${ }^{28}$ A. Kumar, G. Singh, T. Joshi, G.K. Rao, A.K. Singh, and A.M. Biradar, Appl. Phys. Lett. 100, 54102 (2012).

${ }^{29}$ A. Mertelj, D. Lisjak, M. Drofenik, and M. Čopič, Nature 504, 237 (2013).

${ }^{30}$ M. Shuai, A. Klittnick, Y. Shen, G.P. Smith, M.R. Tuchband, C. Zhu, R.G. Petschek, A. Mertelj, D. Lisjak, M. Čopič, J.E. Maclennan, M.A. Glaser, and N.A. Clark, Nat. Commun. 7, 10394 (2016).

${ }^{31}$ R. Basu, Phys. Rev. E 89, 22508 (2014).

${ }^{32}$ R. Kempaiah, Y. Liu, Z. Nie, and R. Basu, Appl. Phys. Lett. 108, 83105 (2016).

33 S. Al-Zangana, M. Iliut, M. Turner, A. Vijayaraghavan, and I. Dierking, Adv. Opt. Mater. 4, 1541 (2016).

${ }^{34}$ S. Al-Zangana, M. Iliut, G. Boran, M. Turner, A. Vijayaraghavan, and I. Dierking, Sci. Rep. 6, 31885 (2016).

${ }^{35}$ B.R. Ratna and R. Shashidhar, Pramana 6, 278 (1976).

${ }^{36}$ W. Gao, Graphene Oxide (Springer International Publishing, Cham, 2015).

${ }^{37}$ P. Debye, Polar Molecules (Chemical Catalogue Company, New York, 1929).

${ }^{38}$ K.S. Cole and R.H. Cole, J. Chem. Phys. 9, 341 (1941).

${ }^{39}$ K.S. Cole and R.H. Cole, J. Chem. Phys. 10, 98 (1942).

${ }^{40}$ D.W. Davidson and R.H. Cole, J. Chem. Phys. 18, 1417 (1950).

${ }^{41}$ D.W. Davidson and R.H. Cole, J. Chem. Phys. 19, 1484 (1951).

${ }^{42}$ Lev M. Blinov, Structure and Properties of Liquid Crystals (Springer, Dordrecht, 2011).

${ }^{43}$ K. Miyasato, S. Abe, H. Takezoe, A. Fukuda, and E. Kuze, Jpn. J. Appl. Phys. 22, L661 (1983). 
Publishiftga. Pinczuk, W. Taylor, and E. Burstein, Solid State Commun. 5, 429 (1967).

${ }^{45}$ A. Scalabrin, A.S. Chaves, D.S. Shim, and S.P.S. Porto, Phys. Status Solidi 79, 731 (1977).

${ }^{46}$ K. Suzuki and K. Kijima, J. Alloys Compd. 419, 234 (2006).

${ }^{47}$ M.B. Smith, K. Page, T. Siegrist, P.L. Redmond, E.C. Walter, R. Seshadri, L.E. Brus, and M.L. Steigerwald, J. Am. Chem. Soc. 130, 6955 (2008).

${ }^{48}$ M. Mitov, C. Bourgerette, and F. de Guerville, J. Phys. Condens. Matter 16, S1981 (2004).

${ }^{49}$ S.A. Paniagua, Y. Kim, K. Henry, R. Kumar, J.W. Perry, and S.R. Marder, ACS Appl. Mater. Interfaces 6, 3477 (2014).

${ }^{50}$ I. Dierking, Adv. Funct. Mater. 14, 883 (2004).

${ }^{51}$ I. Dierking, Liq. Cryst. Today 21, 54 (2012).

${ }^{52}$ F. Li, O. Buchnev, C. Cheon, A. Glushchenko, V. Reshetnyak, Y. Reznikov, T. Sluckin, and J. West, Phys. Rev. Lett. 97, 147801 (2006).

${ }^{53}$ R. Basu and A. Garvey, Appl. Phys. Lett. 105, 151905 (2014).

${ }^{54}$ V.Y. Reshetnyak, S.M. Shelestiuk, and T.J. Sluckin, Mol Cryst. Liq. Cryst. 454, 201 (2006).

${ }^{55}$ R.K. Shukla, D.R. Evans, and W. Haase, Ferroelectrics 500, 141 (2016).

${ }^{56}$ L.M. Blinov and V.G. Chigrinov, Electrooptic Effects in Liquid Crystal Materials (springer, New York, 1994).

${ }^{57}$ E. Jakeman and E.P. Raynes, Phys. Lett. A 39, 69 (1972).

${ }^{58}$ H.-H. Liang, Y.-Z. Xiao, F.-J. Hśh, C.-C. Wu, and J.-Y. Lee, Liq. Cryst. 37, 255 (2010).

${ }^{59}$ T. Sasaki, A. Katsuragi, O. Mochizuki, and Y. Nakazawa, J. Phys. Chem. B 107, 7659 (2003).

${ }^{60}$ P. Archer and I. Dierking, J. Opt. A Pure Appl. Opt. 11, 24022 (2009).

${ }^{61}$ D.M. Potukuchi, a. K. George, C. Carboni, J. Naciri, and S.H. Al-Harthi, Ferroelectrics 300, 79 (2004).

${ }^{62}$ R.K. Shukla, J. Mirzaei, A. Sharma, D. Hofmann, T. Hegmann, and W. Haase, RSC Adv. 5, 34491 (2015).

${ }^{63}$ A. Kumar, J. Prakash, A. Choudhary, and A.M. Biradar, J. Appl. Phys. 105, 124101 (2009).

${ }^{64}$ M. Petit, in Polymerization (InTech, 2012).

65 P. Malik, A. Chaudhary, R. Mehra, and K.K. Raina, Mol. Cryst. Liq. Cryst. 541, 243/[481] (2011).

${ }^{66}$ T. Carlsson, B. Žekš, C. Filipič, and A. Levstik, Phys. Rev. A 42, 877 (1990).

${ }^{67}$ S. Kimura, S. Nishiyama, Y. Ouchi, H. Takezoe, and A. Fukuda, Jpn. J. Appl. Phys. 26, L255 (1987).

${ }^{68}$ F.R. Germany, 3, 469 (1988).

69 S.T. Lagerwall, Ferroelectric and Antiferroelectric Liquid Crystals (WILEY-VCH Verlag 33 
Publishiøgob, Weinheim-Germany, 1999).

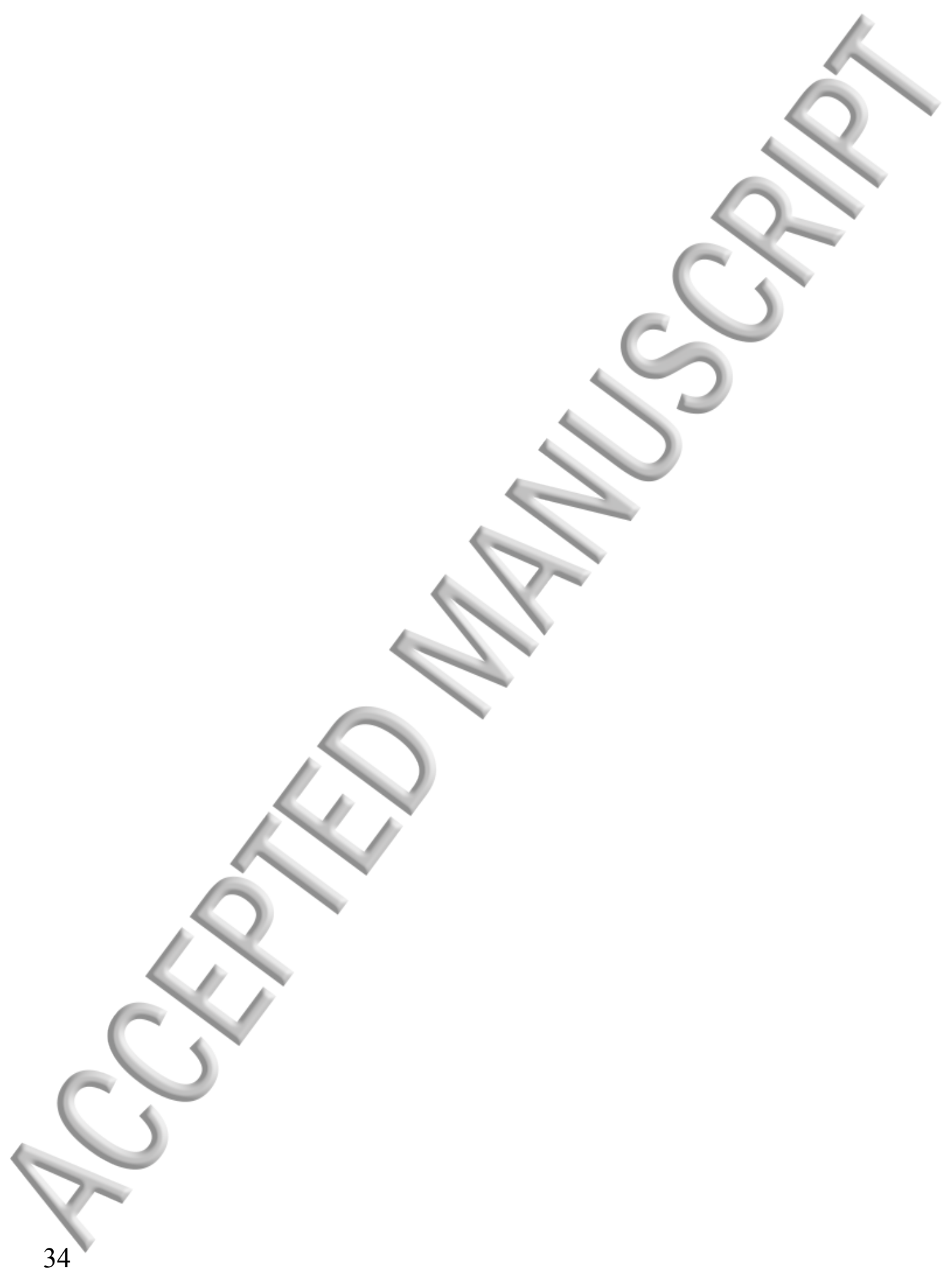







BTO1

(a)
$200 \mathrm{~nm}$
BTO2

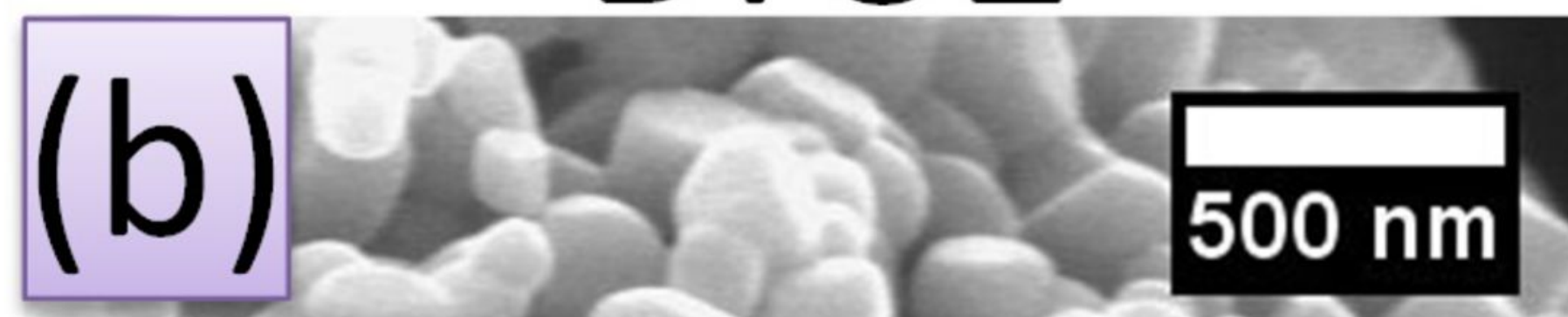

$500 \mathrm{~nm}$

$\left(\mathrm{f}(\mathrm{c}) \mathrm{40}^{40}\right.$

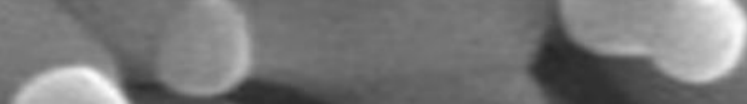
BTO1 $=(80 \pm 20) \mathrm{nm}$
BTO2 $=(240 \pm 80) \mathrm{nm}$

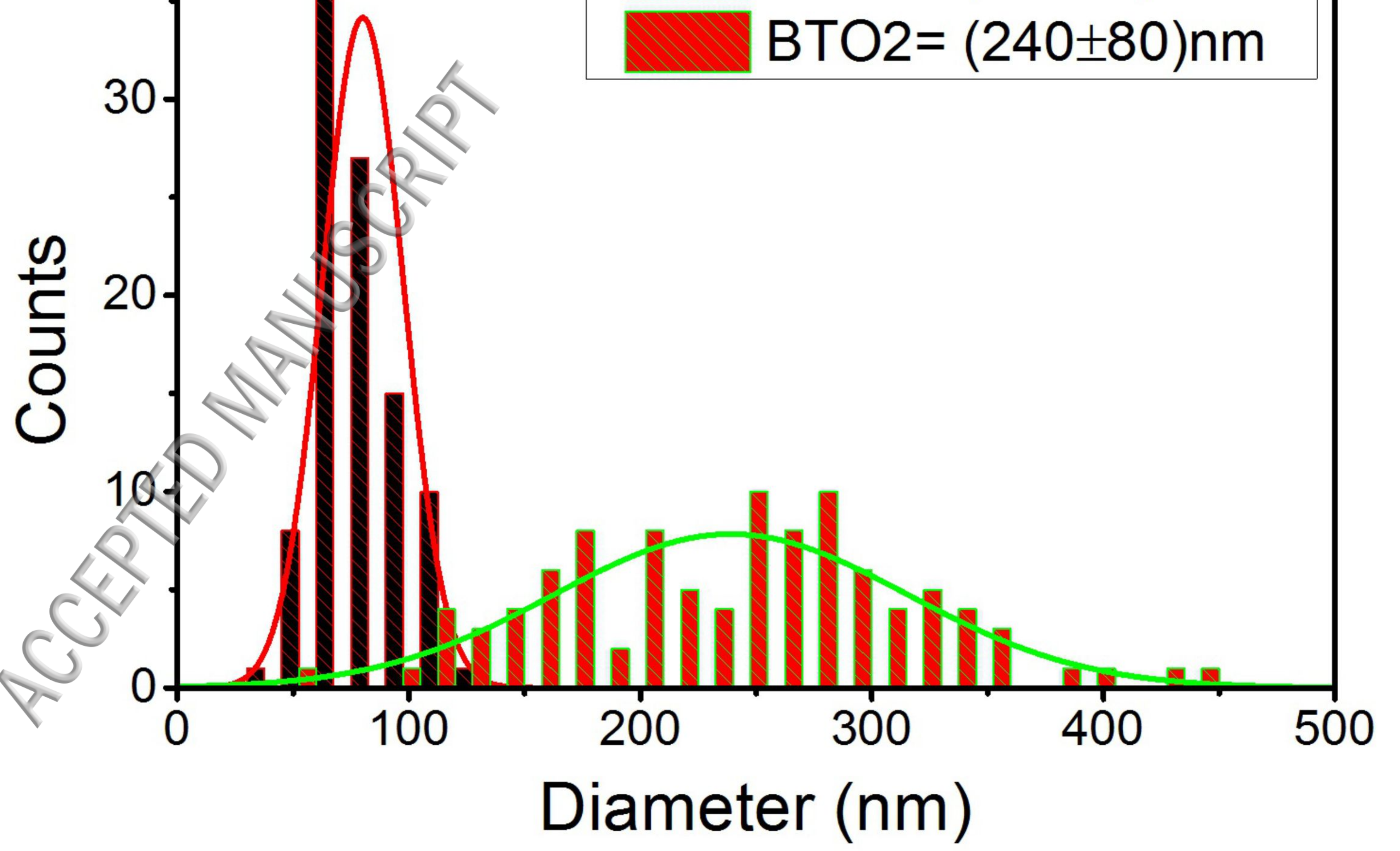


(b)

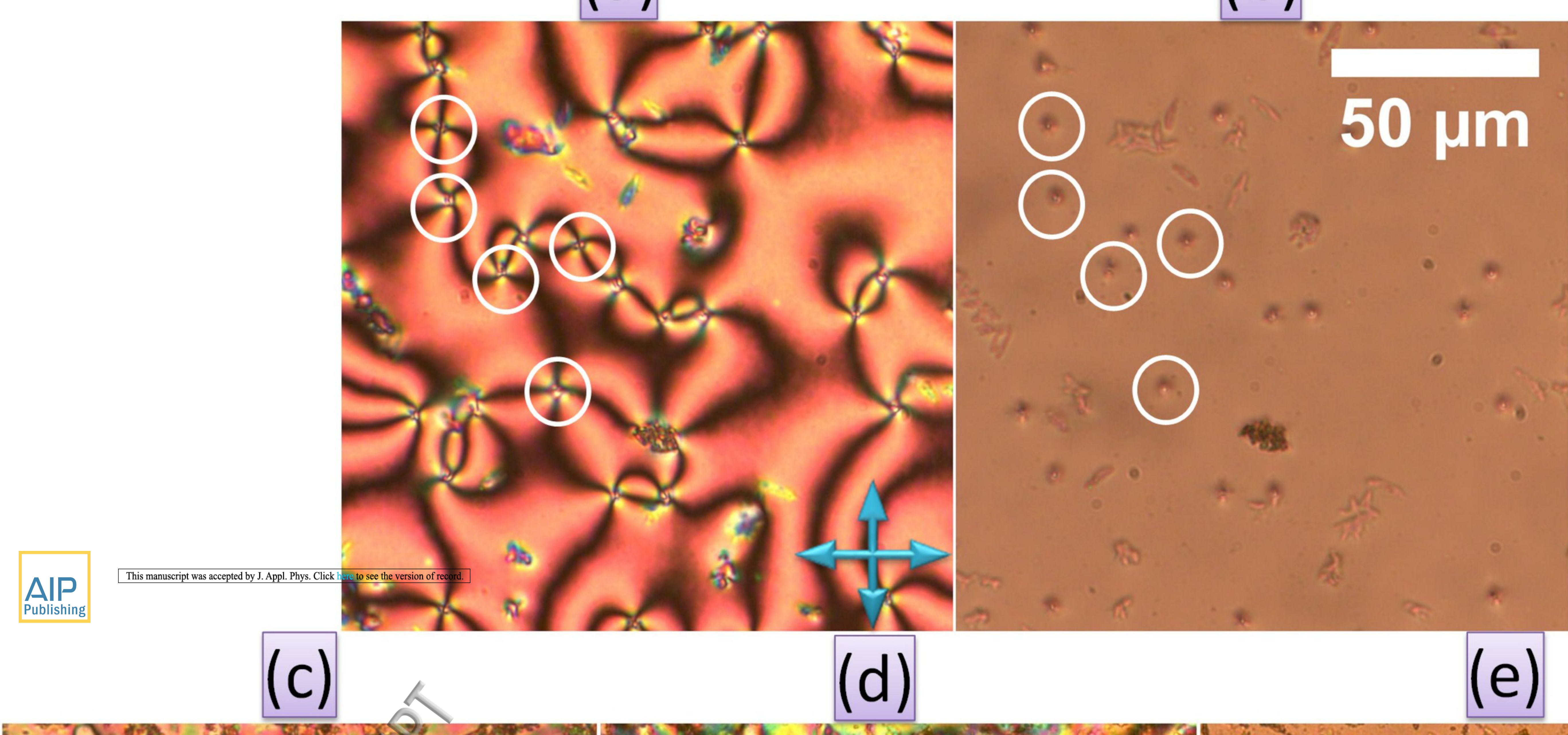

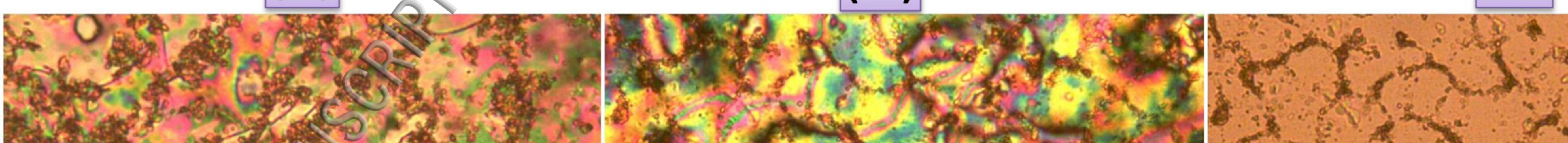

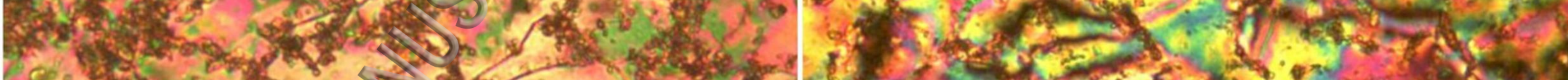

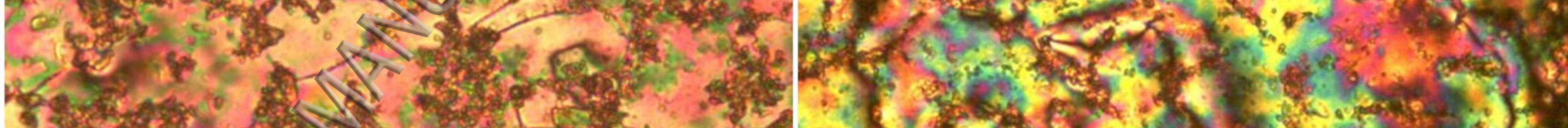
3.

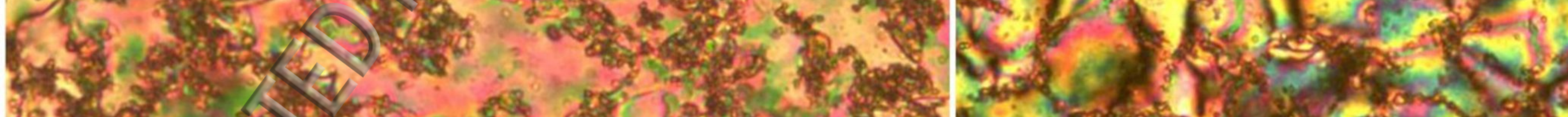
H.

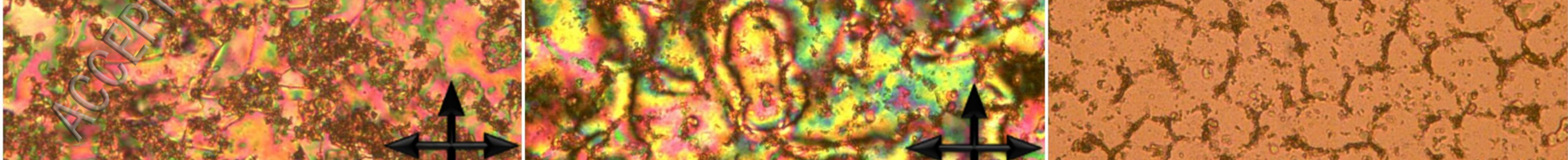

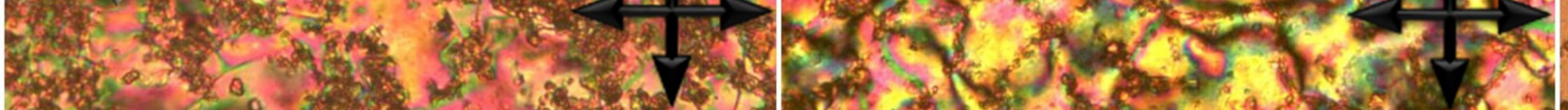

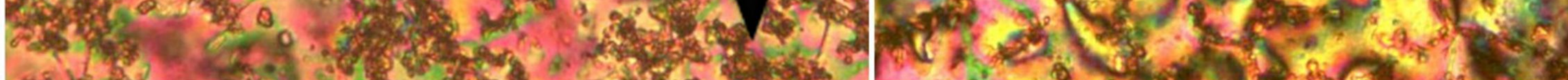









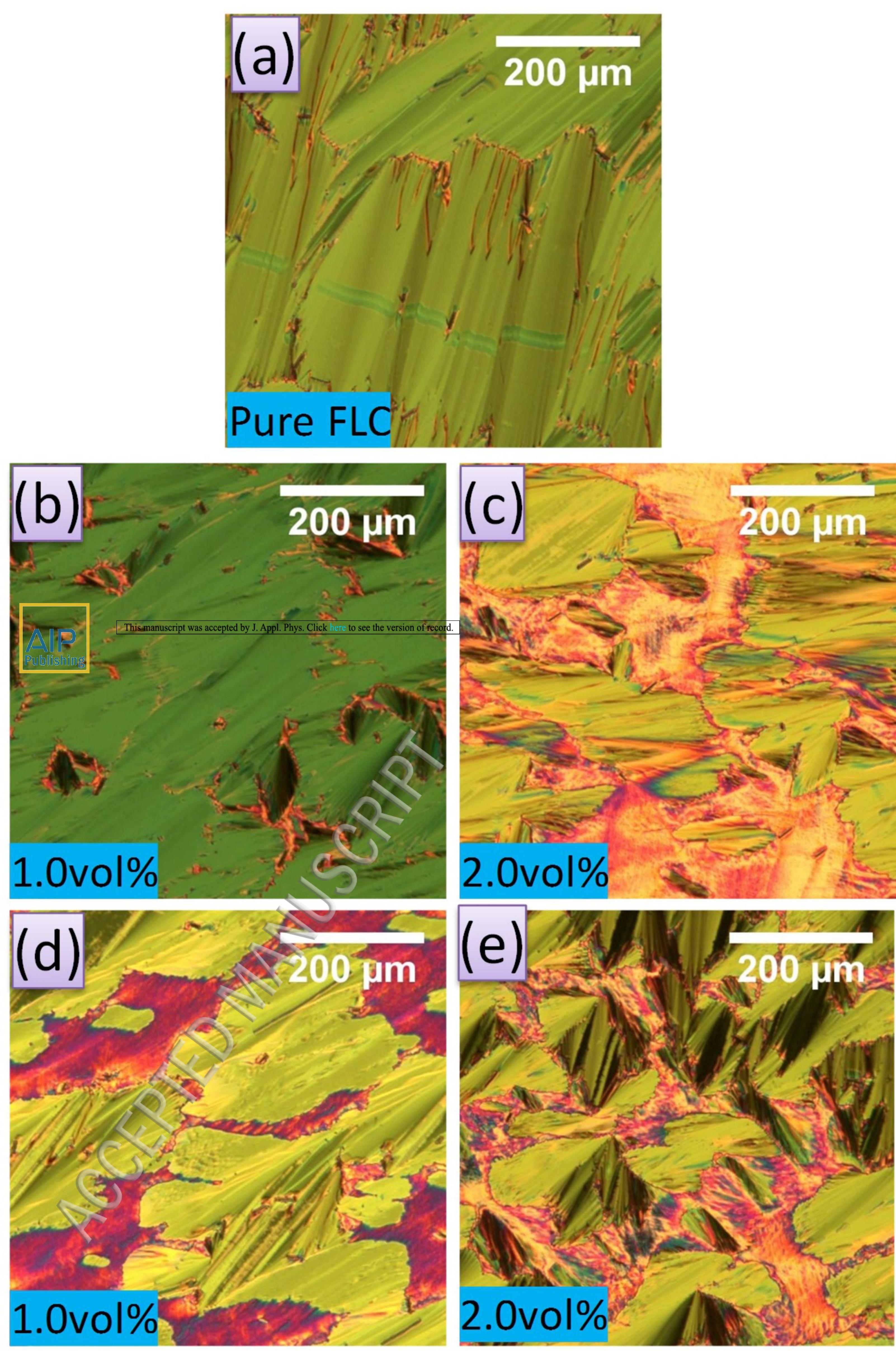


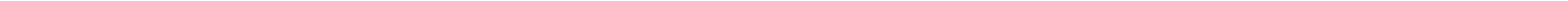




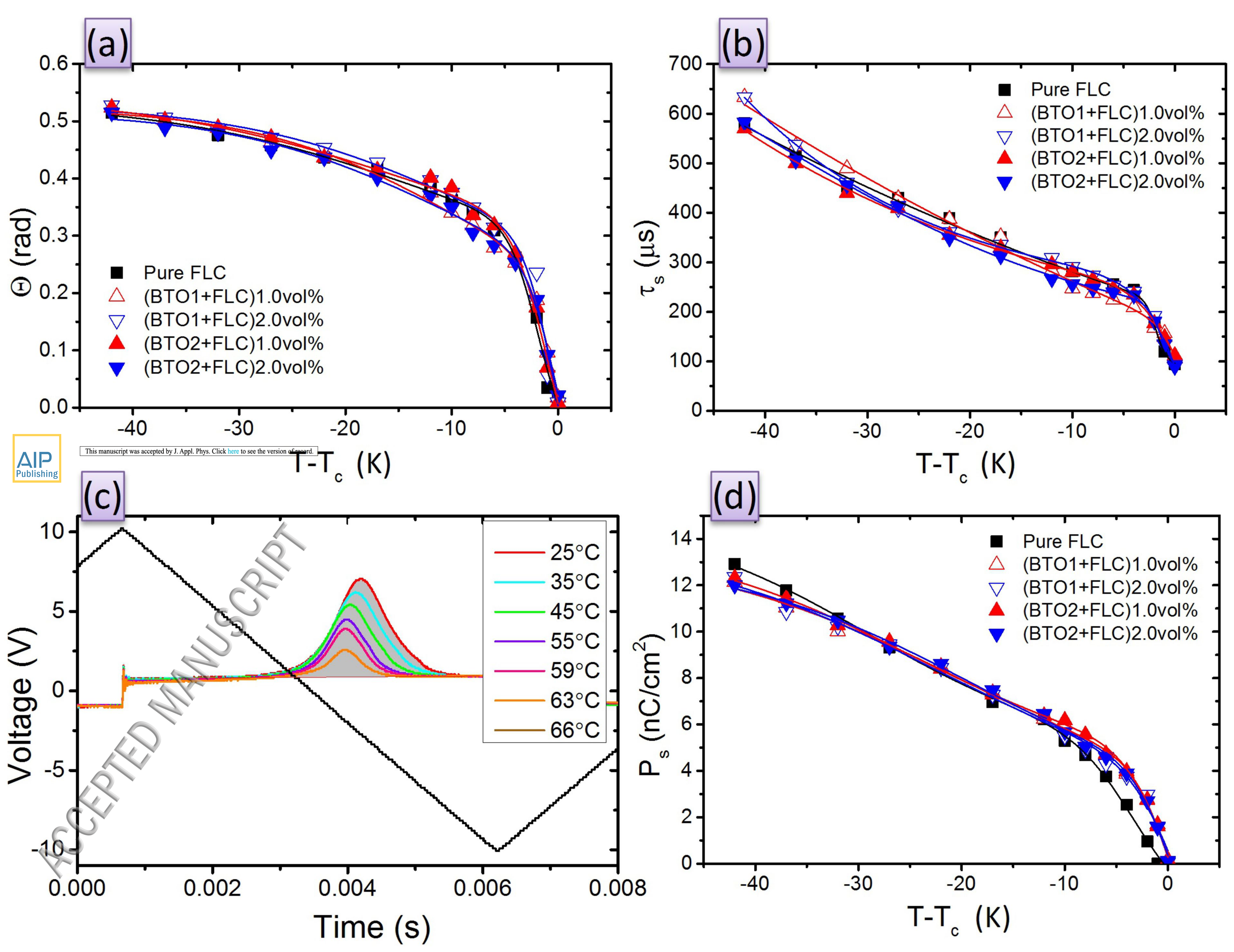




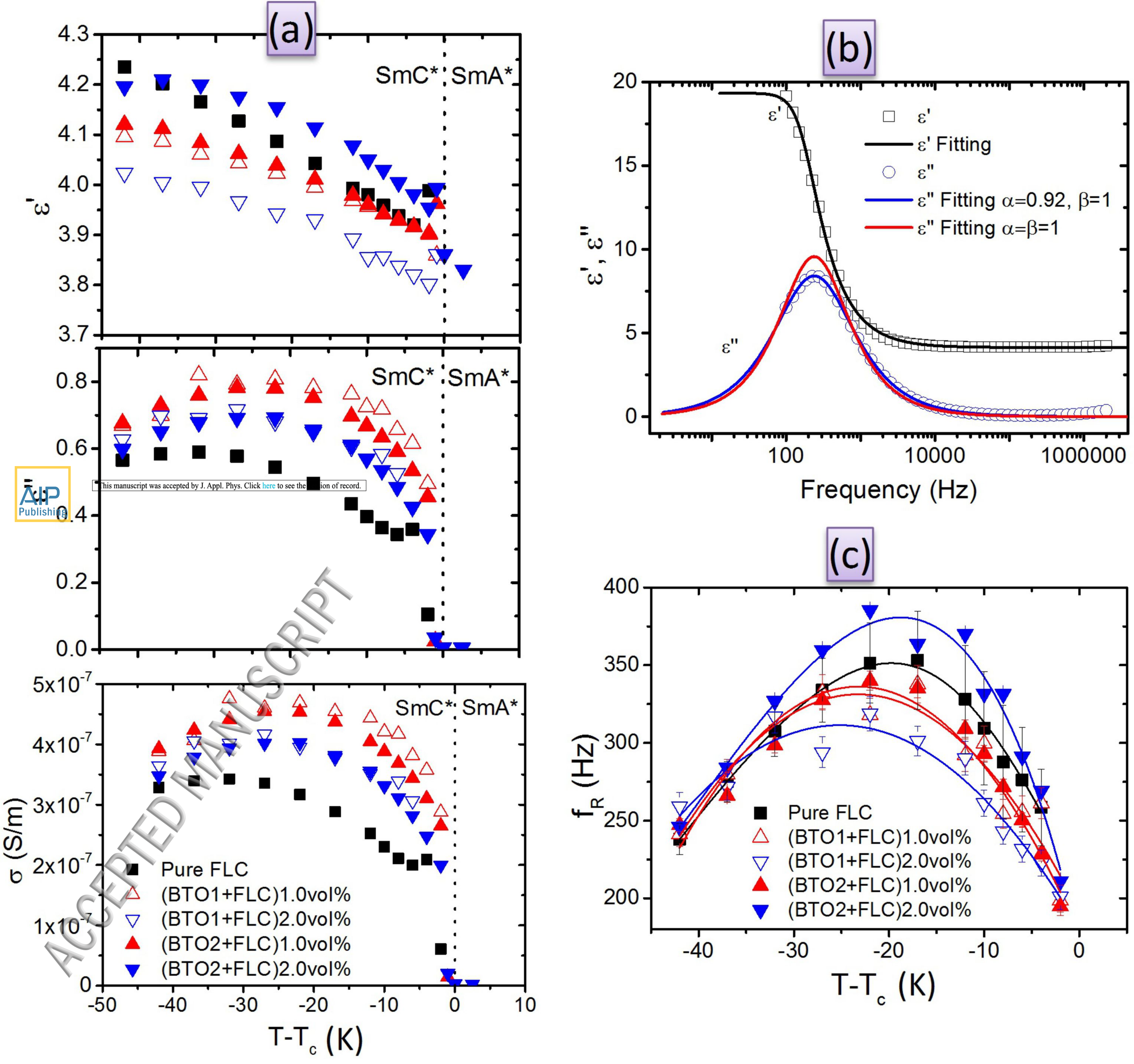



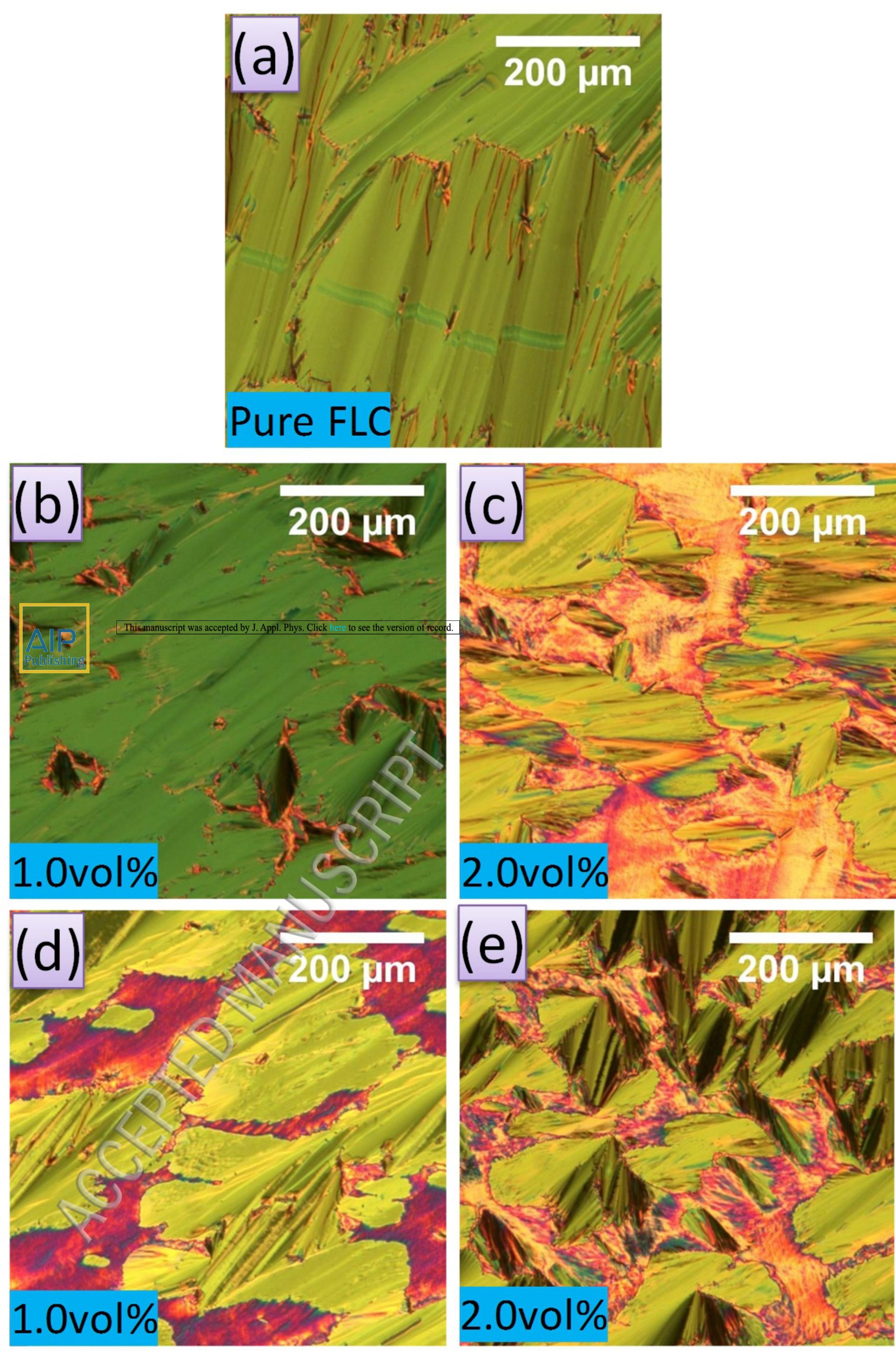